\title{
A probabilistic generative model for semi-supervised training of coarse-grained surrogates and enforcing physical constraints through virtual observables
}

\author{
Maximilian Rixner ${ }^{\mathrm{a}}$, Phaedon-Stelios Koutsourelakis ${ }^{\mathrm{a}, *}$ \\ ${ }^{a}$ Professorship of Continuum Mechanics, Technical University of Munich
}

\begin{abstract}
The data-centric construction of inexpensive surrogates for fine-grained, physical models has been at the forefront of computational physics due to its significant utility in many-query tasks such as uncertainty quantification. Recent efforts have taken advantage of the enabling technologies from the field of machine learning (e.g. deep neural networks) in combination with simulation data. While such strategies have shown promise even in higher-dimensional problems, they generally require large amounts of training data even though the construction of surrogates is by definition a Small Data problem. Rather than employing data-based loss functions, it has been proposed to make use of the governing equations (in the simplest case at collocation points) in order to imbue domain knowledge in the training of the otherwise black-box-like interpolators. The present paper provides a flexible, probabilistic framework that accounts for physical structure and information both in the training objectives as well as in the surrogate model itself. We advocate a probabilistic (Bayesian) model in which equalities that are available from the physics (e.g. residuals, conservation laws) can be introduced as virtual observables and can provide additional information through the likelihood. We further advocate a generative model i.e. one that attempts to learn the joint density of inputs and outputs that is capable of making use of unlabeled data (i.e. only inputs) in a semi-supervised fashion in order to promote the discovery of lower-dimensional embeddings which are nevertheless predictive of the fine-grained model's output.
\end{abstract}

Keywords: probabilistic machine learning, virtual observables, high-dimensional surrogates, semi-supervised learning, unlabeled data

${ }^{*}$ Corresponding author

Email addresses: maximilian.rixner@tum.de (Maximilian Rixner), p.s.koutsourelakis@tum.de (Phaedon-Stelios Koutsourelakis) 


\section{Introduction}

The complexity and cost of many models in computational physics, necessitates the development of less expensive surrogates (or coarse-grained/reduced-order models) that provide insight but more importantly retain predictive accuracy and can enable many-query applications such as uncertainty quantification. The difficulty of such problems is amplified in the high-dimensional setting i.e. when the number of input-output (random) variables is large as in most cases of practical interest. Data-based surrogates must also be capable of dealing with the scarcity of training data (Koutsourelakis et al., 2016). Unlike recent successes in statistical/machine learning, and supervised learning in particular, which in large part have been enabled by large datasets (and the computational means to leverage them), the acquisition of data, i.e. pairs of input-outputs, is the most expensive task and the reduction of their number, the primary objective of surrogate development.

Another critical challenge stems from the nature of the physical models themselves. Their primary utility arises from their ability to distill apparent complexity and high-dimensional descriptions into much fewer, essential variables and the relations between them, which can in turn be used to make accurate predictions under a variety of settings (e.g. different boundary/initial conditions, right-hand-sides etc). This robustness of physical models as well as their ability to operate under extrapolative conditions is not a property shared by black-box statistical surrogates, which in most cases are used in interpolative settings.

We argue that to overcome these challenges, domain knowledge i.e. information about the underlying physical/mathematical structure of the problem, must be injected into the surrogates constructed (Marcus and Davis, 2019). While this prior physical knowledge is generally plentiful and eloquently reflected in the governing equations, it is not necessarily obvious neither how to to mine it nor how to automatically combine it with the data-based learning objectives, especially in a probabilistic setting (Stewart and Ermon, 2017). We believe that a probabilistic framework provides a superior setting for such problems as it is capable of quantifying predictive uncertainties which are unavoidable when any sort of model/dimensionality reduction is pursued and when the surrogate model is learned from finite (and hopefully, small) data (Koutsourelakis, 2007).

The development of surrogates for the purposes of uncertainty quantification in the context of continuum thermodynamics where pertinent models are based on PDEs and ODEs has a long history. Some of the most well-studied methods have been based on (generalized) Polynomial Chaos expansions (gPC) (Ghanem and Spanos, 1991; Xiu and Karniadakis, 2002a) which have gained popularity due to the emergence of data-based, non-intrusive, sparse-grid stochastic collocation approaches (Xiu and Hesthaven, 2005; Ma and Zabaras, 2009; Lin and Tartakovsky, 2009). These approaches typically struggle with high-dimensional stochastic inputs, as is the case e.g. when random heterogeneous media (Torquato and Lu, 1993) are considered.

Another strategy for the construction of inexpensive surrogates is offered by reduced-basis (RB) methods (Hesthaven et al., 2016; Quarteroni et al., 2016) where, based on a small set of "snapshots" i.e. input-output pairs, the solution space's dimensionality is reduced by projection onto the principal directions. Classical formulations rely on (Petrov-)Galerkin projections (Rowley et al., 2004) for finding the associated coefficients, but recently several efforts have been directed towards unsupervised and supervised learning strategies (Guo and Hesthaven, 2018a; Hesthaven and Ubbiali, 2018; Kani and Elsheikh, 2017; Wang et al., 2020). Apart from issues of efficiency and stability, RB approaches in their standard form are generally treated in a non-Bayesian way and therefore only yield point estimates instead of full predictive poste- 
rior distributions. Furthermore, since scalar- or vector- or matrix-valued quantities need to be learned as a function of the parametric input in the offline phase, they are also challenged by the high-dimensions/small-data setting considered (Lee and Carlberg, 2020).

A more recent trend is to view surrogate modeling as a supervised learning problem and employ pertinent statistical learning tools, e.g. Gaussian Process (GP) regression (Rasmussen and Williams, 2006; Bilionis et al., 2013a; Bilionis and Zabaras, 2017) and which can frequently provide closed-form predictive distributions. Although several advances have been made towards multi-fidelity data fusion (O'Hagan and Kennedy, 2000; Koutsourelakis, 2009; Raissi et al., 2017a; Perdikaris et al., 2015) and incorporation of physical information (Yang et al., 2018; Lee et al., 2018; Tipireddy and Tartakovsky, 2018; Guo and Hesthaven, 2018b) via Gaussian Processes, their performance and scaling with stochastic input dimension remains one of the main challenges for GP models. In the context of supervised learning, deep neural networks (DNNs) (LeCun et al., 2015; Goodfellow et al., 2016) have found their way into surrogate modeling of complex computer codes (Han et al., 2018; Zhu and Zabaras, 2018; Mo et al., 2018; Sirignano and Spiliopoulos, 2018; E and Yu, 2018). One of the most promising developments in the adaptation of such tools for physical modeling are physics-informed neural networks (Raissi et al., 2017b; Raissi and Karniadakis, 2018; Raissi et al., 2019; Yang and Perdikaris, 2019) which are trained by minimizing a loss function augmented by the residuals of the governing equations (Lagaris et al., 2000). Physical knowledge in training DNNs has also been introduced in the form of residuals in (Raissi et al., 2017b; Kani and Elsheikh, 2017; Nabian and Meidani, 2018; Beck et al., 2019; Karumuri et al., 2020; Khodayi-Mehr and Zavlanos, 2019) whereas in (Zhu et al., 2019), a Boltzmann-type density containing physics-based functionals or residuals were employed as the target for the associated learning problem. Recent reviews of the use of various machine learning models, and in particular deep neural networks, for the solution of problems in computational physics, including the development of surrogates, can be found in (Frank et al., 2020; Willard et al., 2020). Therein the difficulty of the task of incorporating physical, domainknowledge into machine learning objectives and tools (Mattheakis et al., 2020; Magiera et al., 2020 ) is detailed as well as the scarcity of probabilistic approaches in the context of such tasks.

In contrast to the majority of the efforts detailed above, our goal is not to develop approximations to the PDE-solution but to capture its dependence on high-dimensional parametric vectors. For that purpose we consider as our reference model a discretized version of the PDE which is assumed to provide sufficiently accurate resolution (we refer to this as the Fine-Grained Model (FGM)). Furthermore, we wish to differentiate our work from applications of machine learning in problems where the underlying governing equations themselves are assumed unknown and one aims to identify them from data (Brunton et al., 2016; Long et al., 2017; Felsberger and Koutsourelakis, 2019). While a component of our model makes use of a (discretized) coarse-grained model, its form is in this work prescribed.

We propose overcoming the aforementioned challenges by introducing a novel, generative probabilistic model that is capable of exploiting labeled (i.e. input-output pairs) and unlabeled (i.e. only inputs) data in discovering lower-dimensional embeddings and identifying the right surrogate model-structure (section 2). More importantly, we propose augmenting the aforementioned data by injecting domain knowledge in a principled manner in the probabilistic models employed. In particular, such physical/mathematical knowledge is incorporated:

- in the learning objectives (section 2.2) through the novel notion of virtual observables (Kaltenbach and Koutsourelakis, 2019). We demonstrate how various types of information in the form of (non)linear equalities/constraints as well as minimizing functionals can be 
introduced in the likelihood terms.

- in an appropriately selected coarse-grained model (CGM, section 2.3) which through coarsened or reduced-physics versions of the full-order model provides an integral component of the proposed surrogate.

We complement the aforementioned elements with a integrated, supervised dimensionality reduction scheme which can distill lower-dimensional features of the high-dimensional input that are most predictive of the high-dimensional output and which is trained simultaneously with the other components by making use of (un)labeled data and virtual observables. We employ Stochastic Variational Inference procedures for training the proposed model (section 2.5), which upon completion yields a probabilistic surrogate that not only produces point estimates of the high-dimensional output but can quantify the predictive uncertainty associated with this task (section 2.6). We assess the predictive performance of the proposed framework in section 3 where we demonstrate that unlabeled data and virtual observables can lead to significant improvements in its generalization accuracy and can reduce the number of labeled data (i.e. input-outputs pairs) to a few tens. Furthermore, we illustrate the model's ability to [perform equally well under interpolative and extrapolative conditions i.e. under boundary conditions seen or not seen during training. We finally demonstrate its benefits in an uncertainty propagation problem and discuss possible extensions in section 4 .

\section{Methodology}

We consider steady-state physical processes modeled by a partial differential equation:

$$
\mathcal{L}(u(s) ; \boldsymbol{x})=0, \quad s \in \Omega
$$

over the physical domain $\Omega \subset \mathbb{R}^{d}$. The differential operator $\mathcal{L}$ depends on the random parameters $\boldsymbol{x} \in \mathbb{R}^{d_{x}}$ and so does the solution of the PDE $u(s)$. We denote by $\boldsymbol{y} \in \mathbb{R}^{d_{y}}$ discretized versions of the latter and by $\boldsymbol{y}(\boldsymbol{x})$ the input-output map implied by any of the usual PDE-discretization schemes. The governing equations are complemented by boundary conditions which might partially or completely depend on the parameters $\boldsymbol{x}$. We refer to this discretized model as finegrained model (FGM). We are interested in FGMs that are computationally demanding, i.e. the number of forward model runs determines the cost of the analysis task of interest (e.g. forward or backward uncertainty propagation, optimization). Furthermore, the problems of interest are highdimensional, i.e. $d_{x}, d_{y}>>1$, as in most cases of practical interest. Our goal is to construct a surrogate with the least possible labeled data $N_{l}$ i.e. input-output pairs $\mathcal{D}_{l}=\left\{\boldsymbol{x}^{(i)}, \boldsymbol{y}^{\left(i_{i l}\right)}=\boldsymbol{y}\left(\boldsymbol{x}^{\left(i_{l}\right)}\right)\right\}_{i_{l}=1}^{N_{l}}$.

Several probabilistic discriminative models i.e. models that attempt to learn $p(\boldsymbol{y} \mid \boldsymbol{x})$ have been proposed e.g. using Gaussian Processes Bilionis et al. (2013b), Polynomial Chaos (Xiu, 2010; Xiu and Karniadakis, 2002b) or more recently using Deep Neural Networks (Raissi et al., 2019; Zhu and Zabaras, 2018; Nabian and Meidani, 2018; Khodayi-Mehr and Zavlanos, 2019). It is clear that in the Small Data setting, such attempts can be generalizable only if the problem is amenable to dimensionality reductions i.e. a lower-dimensional set of features of $\boldsymbol{x}$ are predictive of $\boldsymbol{y}$ and/or the latter itself lives in a lower-dimensional manifold. The simultaneous discovery of 
such lower-dimensional embeddings through a latent variable model was demonstrated in Grigo and Koutsourelakis $(2019 a, b)$ where the sought density $p(\boldsymbol{y} \mid \boldsymbol{x})$ was approximated by:

$$
p_{\theta}(\boldsymbol{y} \mid \boldsymbol{x})=\int p_{\theta}(\boldsymbol{y} \mid z) p_{\theta}(z \mid x) d x
$$

with $\boldsymbol{\theta}$ being the trainable parameters of the model. The variables $z \in \mathbb{R}^{d_{z}}$ represent the lowerdimensional (i.e. $d_{z}<<d_{x}, d_{y}$ ) information bottleneck between inputs and outputs. In the aforementioned works, these have been associated with a lower-fidelity physical model and have been identified in the presence of Small Data using Sparse Bayesian learning from a large vocabulary of physically-motivated features of $\boldsymbol{x}$.

\subsection{Generative Model}

The first novel contribution of this work is the use of a generative model i.e. one that attempts to approximate the joint density $p(\boldsymbol{x}, \boldsymbol{y})$ and which can subsequently be used by conditioning on $\boldsymbol{x}$ for predictive purposes. Such a model has the capability of ingesting unlabeled data (i.e. only inputs) $\mathcal{D}_{u}=\left\{\boldsymbol{x}^{\left(i_{u}\right)}\right\}_{i_{u}=1}^{N_{u}}$ and therefore enable semi-supervised learning. This in turn allows the use of the information provided by the inexpensive (and potentially large) dataset $\mathcal{D}_{u}$ which can reduce the dependence on the expensive labeled data Chapelle et al. (2009); Kingma et al. (2014). In particular, we propose a model that performs supervised dimensionality reduction of $\boldsymbol{x}$ and $\boldsymbol{y}$ Yu et al. (2006) and for each labeled pair $i_{l}$ in $\mathcal{D}_{l}$ assigns a likelihood:

$$
p_{\boldsymbol{\theta}}\left(\boldsymbol{x}^{\left(i_{l}\right)}, \boldsymbol{y}^{\left(i_{l}\right)}\right)=\int p_{\boldsymbol{\theta}}\left(\boldsymbol{y}^{\left(i_{l}\right)} \mid \boldsymbol{z}^{\left(i_{l}\right)}\right) p_{\boldsymbol{\theta}}\left(\boldsymbol{x}^{\left(i_{l}\right)} \mid z^{\left(i_{l}\right)}\right) p_{\boldsymbol{\theta}}\left(z^{\left(i_{l}\right)}\right) d \boldsymbol{z}^{\left(i_{l}\right)}
$$

We denote again with $\boldsymbol{\theta}$ any tunable model parameters although these are in general different from the ones in Equation (2). The unobserved variables $\boldsymbol{z}$ play the role of latent generators of $\boldsymbol{x}$ and $\boldsymbol{y}$ which constitute them (conditionally) independent. We specify the form of the aforementioned densities, their parameterization as well as their training in the sequel. We note that the generative construction adopted provides also a likelihood for each unlabeled datapoint $i_{u}$ in $\mathcal{D}_{u}$ as follows:

$$
p_{\theta}\left(\boldsymbol{x}^{\left(i_{u}\right)}\right)=\int p_{\boldsymbol{\theta}}\left(\boldsymbol{x}^{\left(i_{u}\right)} \mid \boldsymbol{z}^{\left(i_{u}\right)}\right) p_{\boldsymbol{\theta}}\left(z^{\left(i_{u}\right)}\right) d \boldsymbol{z}^{\left(i_{u}\right)}
$$

Furthermore, for predictive purposes, the posterior of $z$ for a new $\boldsymbol{x}$ i.e. $p_{\theta}(z \mid \boldsymbol{x}) \propto p_{\boldsymbol{\theta}}(\boldsymbol{x} \mid z) p_{\theta}(z)$ can be used in order to compute:

$$
p_{\theta}(\boldsymbol{y} \mid \boldsymbol{x})=\int p_{\theta}(\boldsymbol{y}, \boldsymbol{z} \mid \boldsymbol{x}) d z=\int p_{\theta}(\boldsymbol{y} \mid \boldsymbol{z}) p_{\theta}(z \mid \boldsymbol{x}) d z
$$

i.e. the predictive posterior on the corresponding output $y$. Figures $1 \mathrm{a}$ and $1 \mathrm{~b}$ provide illustrations of the discriminative and generative probabilistic graphical models.

\subsection{Virtual Observables}

The second novelty proposed in this paper pertains to the introduction of domain knowledge as represented in the governing equation (Equation (1)) into the learning objectives. We would like the training process not to rely exclusively on unlabeled $\mathcal{D}_{u}$ or labeled $\mathcal{D}_{l}$ data but rather on physical knowledge and information. These can appear in several forms but since we 


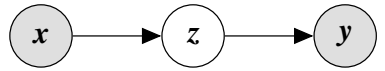

(a) Discriminative model

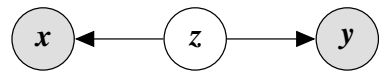

(b) Generative model

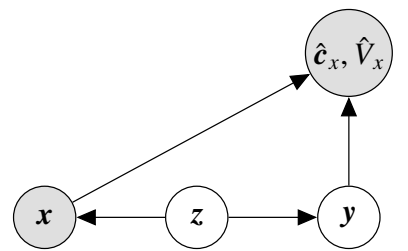

(c) Generative model with virtual observables

Figure 1: Illustration of differences between probabilistic graphical models discussed. a) Discriminative model where the latent variables $\boldsymbol{z}$ encode lower-dimensional features of the input $\boldsymbol{x}$ which are predictive of the output $\boldsymbol{y}, \mathrm{b}$ ) Generative model where $\boldsymbol{z}$ represent latent generators of both input and output, and c) Generative model where (b) is augmented by virtual observables encoding domain knowledge.

are interested in their systematic incorporation we consider here various (in)equalities expressing different types of physical relations between the model-variables. The governing PDE of Equation (1) for example, is a potentially infinite source of information (if one considers that the equality holds at each of the infinite points of the problem domain $\Omega$ ) in contrast to the limited times these governing equations can be solved due to computational expense. While the introduction of such equalities is rather straightforward in deterministic settings in the training loss and has been employed successfully in the context of physics-informed neural networks (PINNs Raissi et al. (2019)), in a probabilistic setting, it has only been achieved for linear ones and in order to approximate the solution of the PDE (not its dependence on input parameters) using Gaussian Processes (Raissi et al., 2017c). In this work, we generalize the type of equalities that we consider by including nonlinear ones as well as demonstrate how other types of information, e.g. that solution is a minimizer of a functional, can be introduced. We discuss below such how these can be incorporated in the learning/inference process and we give specific examples of the forms these take in the numerical illustrations (section 3 ).

Consider first equality constraints, i.e.:

$$
c(y ; x)=0
$$

where $c: \mathbb{R}^{d_{y}} \times \mathbb{R}^{d_{x}} \rightarrow \mathbb{R}^{d_{c}}$. Such equalities can represent residuals of the governing PDE computed e.g. at some collocation points or by employing weighted residuals with appropriate test functions. They might also represent the enforcement of a physical constraint such as a conservation law (e.g. mass, momentum, energy). The only requirement on $c$ imposed by our framework is that they are differentiable functions, a property that will prove crucial in the Variational Inference component (section 2.5). In order to incorporate Equation (6), we introduce an auxiliary variable/vector $\hat{\boldsymbol{c}}_{x}$ which relates to $\boldsymbol{c}$ as follows:

$$
\hat{\boldsymbol{c}}_{x}=\boldsymbol{c}(\boldsymbol{y} ; \boldsymbol{x})+\sigma_{c} \boldsymbol{\epsilon}_{c}, \quad \boldsymbol{\epsilon}_{c} \sim \mathcal{N}(\mathbf{0}, \boldsymbol{I})
$$

We further assume that $\hat{\boldsymbol{c}}_{x}$ is virtually observed and $\hat{\boldsymbol{c}}_{x}=0$. This induces a virtual likelihood $p\left(\hat{\boldsymbol{c}}_{x} \mid \boldsymbol{x}, \boldsymbol{y}\right)$ i.e.:

$$
p\left(\hat{\boldsymbol{c}}_{x}=\mathbf{0} \mid \boldsymbol{x}, \boldsymbol{y}\right) \propto \frac{1}{\sigma_{c}^{d_{c} / 2}} e^{-\frac{1}{2 \sigma_{c}^{2}}|\boldsymbol{c}(\boldsymbol{y} ; \boldsymbol{x})|^{2}}
$$

The parameter $\sigma_{c}$ determines the intensity of the enforcement of the virtual observation and is analogous to the tolerance parameter with which constraints or residuals are enforced in a 
deterministic solvers. In the limit that $\sigma_{c} \rightarrow 0$, the likelihood above degenerates to a Dirac-delta concentrated on the manifold implied by the constraint. We note further that in the context of the generative model one can exploit additional unlabeled data, $\left\{\boldsymbol{x}^{\left(i_{c}\right)}, \hat{\boldsymbol{c}}_{x}^{\left(i_{c}\right)}\right\}$ consisting of pairs of inputs and virtual observables and the likelihood of each such data-pair $i_{c}$ will be:

$$
\begin{aligned}
p_{\boldsymbol{\theta}}\left(\boldsymbol{x}^{\left(i_{c}\right)}, \hat{\boldsymbol{c}}_{x}^{\left(i_{c}\right)}=\mathbf{0}\right) & =\int p_{\boldsymbol{\theta}}\left(\hat{\boldsymbol{c}}_{c}^{\left(i_{c}\right)}, \boldsymbol{y}^{\left(i_{c}\right)}, \boldsymbol{z}^{\left(i_{c}\right)}, \boldsymbol{x}^{\left(i_{c}\right)}\right) d \boldsymbol{y}^{\left(i_{c}\right)} d z^{\left(i_{c}\right)} \\
& =\int p\left(\hat{\boldsymbol{c}}_{x}^{\left(i_{c}\right)}=\mathbf{0} \mid \boldsymbol{y}^{\left(i_{c}\right)}, \boldsymbol{x}^{\left(i_{c}\right)}\right) p_{\boldsymbol{\theta}}\left(\boldsymbol{y}^{\left(i_{c}\right)}, \boldsymbol{z}^{\left(i_{c}\right)}, \boldsymbol{x}^{\left(i_{c}\right)}\right) d \boldsymbol{y}^{\left(i_{c}\right)} d \boldsymbol{z}^{\left(i_{c}\right)} \\
& =\int p\left(\hat{\boldsymbol{c}}_{x}^{\left(i_{c}\right)}=\mathbf{0} \mid \boldsymbol{y}^{\left(i_{c}\right)}, \boldsymbol{x}^{\left(i_{c}\right)}\right) p_{\boldsymbol{\theta}}\left(\boldsymbol{y}^{\left(i_{c}\right)} \mid z^{\left(i_{c}\right)}\right) p_{\boldsymbol{\theta}}\left(\boldsymbol{x}^{\left(i_{c}\right)} \mid \boldsymbol{z}^{\left(i_{c}\right)}\right) p_{\boldsymbol{\theta}}\left(\boldsymbol{z}^{\left(i_{c}\right)}\right) d \boldsymbol{y}^{\left(i_{c}\right)} d \boldsymbol{z}^{\left(i_{c}\right)}
\end{aligned}
$$

We emphasize that in this case, the solution vector $\boldsymbol{y}^{\left(i_{c}\right)}$ (which satisfies the constraint $\boldsymbol{c}\left(\boldsymbol{y}^{\left(i_{c}\right)} ; \boldsymbol{x}^{\left(i_{c}\right)}\right)$ ) is latent and must be inferred. The corresponding graphical model is illustrated in Figure 1c where the virtual observables represent an observed node (Levine, 2018) with $\boldsymbol{y}$ - the solution of the PDE - becoming a latent variable and therefore unknown quantity in this case.

Another type of physical information that can be accommodated with the concept of virtual observables pertains to the variational nature of the associated problem. It is well-known that the solution of most PDEs in computational physics can be written as minimizers of an appropriate functional. Such functionals have served as the foundation of several numerical schemes and appear in various forms, even for irreversible, nonlinear processes (Ortiz and Stainier, 1999; Yang et al., 2006). Various versions of these functionals were incorporated in the machine learning loss functions Khoo et al. (2017) as well as likelihood functions in probabilistic versions Zhu et al. (2019).

Suppose, that the discretized solution vector $\boldsymbol{y}(\boldsymbol{x})$ is obtained as the minimizer of:

$$
\boldsymbol{y}(\boldsymbol{x})=\arg \min _{\boldsymbol{y}} V(\boldsymbol{y} ; \boldsymbol{x})
$$

where $V: \mathbb{R}^{d_{y}} \times \mathbb{R}^{d_{x}} \rightarrow \mathbb{R}$ represents a generalized free energy or potential. Let $V_{\min }(\boldsymbol{x})=$ $\min _{y} V(\boldsymbol{y} ; \boldsymbol{x})$ be the unknown minimum value of $V$ (attained by the solution) for each $\boldsymbol{x}$. We define the new variable $\hat{V}_{x}$ as follows:

$$
\hat{V}_{x}=V(\boldsymbol{y} ; \boldsymbol{x})-V_{\min }(\boldsymbol{x})-\epsilon_{V}, \quad \epsilon_{V} \sim \operatorname{Expon}\left(\beta^{-1}\right)
$$

The random variable $\epsilon_{V}$ is by construction always non-negative and follows an exponential distribution with parameter $\beta^{1}$. We further assume that $\hat{V}_{x}=0$ has been virtually observed which implies a virtual likelihood:

$$
p\left(\hat{V}_{x}=0 \mid \boldsymbol{y}, \boldsymbol{x}\right)=\beta^{-1} e^{-\beta^{-1}\left(V(\boldsymbol{y} ; \boldsymbol{x})-V_{\text {min }}(\boldsymbol{x})\right)}
$$

As it will be become clear in the sequel, the unknown $V_{\min }(\boldsymbol{x})$ does not enter the training of the model. One can deduce from Equation (12) that the smaller $V(\boldsymbol{y} ; \boldsymbol{x})$ is, the higher the corresponding likelihood becomes and the latter is maximized for the $\boldsymbol{y}$ that corresponds to the solution (Equation (10)). Furthermore, the parameter $\beta$ dictates the decay of the likelihood for $V(\boldsymbol{y} ; \boldsymbol{x})>V_{\min }(\boldsymbol{x})$ and in the limit $\beta^{-1} \rightarrow 0$, the likelihood degenerates to a Dirac-delta concentrated at the minimum (i.e. the true solution).

\footnotetext{
${ }^{1} \epsilon_{V}$ can be thought as the probabilistic analogue of a slack variable for the enforcement of inequality constraints in optimization
} 
As in the previous case of the equality constraints, the introduction of these new observables enables the incorporation of the information contained in the discretized functional $V$ in the training of the proposed generative model. In particular, given unlabeled data $\left\{x^{\left(i_{V}\right)}, \hat{V}_{x}^{\left(i_{V}\right)}\right\}$ consisting of pairs of inputs and virtual observables $\hat{V}_{x}$, the likelihood implied by the model for each data-pair $i_{V}$ will be:

$$
\begin{aligned}
p_{\boldsymbol{\theta}}\left(\boldsymbol{x}^{\left(i_{V}\right)}, \hat{V}_{x}^{\left(i_{V}\right)}=0\right) & =\int p_{\boldsymbol{\theta}}\left(\hat{V}_{x}^{\left(i_{V}\right)}=0, \boldsymbol{y}^{\left(i_{V}\right)}, \boldsymbol{z}^{\left(i_{V}\right)}, \boldsymbol{x}^{\left(i_{V}\right)}\right) d \boldsymbol{y}^{\left(i_{V}\right)} d \boldsymbol{z}^{\left(i_{V}\right)} \\
& =\int p\left(\hat{V}_{x}^{\left(i_{V}\right)}=0 \mid \boldsymbol{y}^{\left(i_{V}\right)}, \boldsymbol{x}^{\left(i_{V}\right)}\right) p_{\boldsymbol{\theta}}\left(\boldsymbol{y}^{\left(i_{V}\right)}, \boldsymbol{z}^{\left(i_{V}\right)}, \boldsymbol{x}^{\left(i_{V}\right)}\right) d \boldsymbol{y}^{\left(i_{V}\right)} d \boldsymbol{z}^{\left(i_{V}\right)} \\
& =\int p\left(\hat{V}_{x}^{\left(i_{V}\right)}=0 \mid \boldsymbol{y}^{\left(i_{V}\right)}, \boldsymbol{x}^{\left(i_{V}\right)}\right) p_{\boldsymbol{\theta}}\left(\boldsymbol{y}^{\left(i_{V}\right)} \mid \boldsymbol{z}^{\left(i_{V}\right)}\right) p_{\boldsymbol{\theta}}\left(\boldsymbol{x}^{\left(i_{V}\right)} \mid \boldsymbol{z}^{\left(i_{V}\right)}\right) p_{\boldsymbol{\theta}}\left(\boldsymbol{z}^{\left(i_{V}\right)}\right) d \boldsymbol{y}^{\left(i_{V}\right)} d \boldsymbol{z}^{\left(i_{V}\right)}
\end{aligned}
$$

As in Equation (9), the solution vector $\boldsymbol{y}^{\left(i_{V}\right)}$ (which minimizes $V\left(\boldsymbol{y} ; \boldsymbol{x}^{\left(i_{V}\right)}\right)$ is latent and must be inferred.

To make our notation independent of specific choices in the remainder we will introduce as a general notation a dataset of virtual observables $\mathcal{D}_{O}=\left\{\boldsymbol{x}^{\left(i_{O}\right)}, \hat{\boldsymbol{o}}^{\left(i_{O}\right)}\right\}_{i=1}^{N_{O}}$, where $\boldsymbol{x}^{\left(i_{O}\right)}$ denotes a input query point and the corresponding $\hat{\boldsymbol{o}}^{\left(i_{0}\right)} \in \mathbb{R}^{M}$ comprises the corresponding virtually observed values. Without loss of generality, we we assume that we enforce the same number of $M$ constraints at every point (this assumption can easily be relaxed). Parameters that govern how rigidly the constrains are enforced - such as $\sigma_{c}^{-1}$ or $\beta$ - are denoted summarily by $\tau$; in the more general case, different constraints can be enforced to varying degrees, i.e. $\tau$ can comprise several precision-type parameters and may be a set instead of a scalar. We refer to each $\boldsymbol{x}^{\left(i_{o}\right)}$ appearing in $\mathcal{D}_{O}$ as input query points to emphasize that in the general case the corresponding solution of the PDE $\boldsymbol{y}(\boldsymbol{x})$ is not observed/known, and we only query certain information from the underlying physics (any equality or inequality constraint implies a certain amount of information about the underlying physics which can be exploited to inform the surrogate and to reduce epistemic uncertainty). The introduction of virtual observables implies that the plausibility of each model contained within the hypothesis space of the generative model $p_{\boldsymbol{\theta}}(\boldsymbol{y}, \boldsymbol{x})$ is scored not only according to its performance on unlabeled and labeled data, but also with respect to the (in)equalities that these represent.

\subsection{Physics-inspired structure for surrogate}

The third contribution of the paper in the direction of imbuing physical knowledge into the machine learning framework pertains to the meaning of the latent variables $z$ and the density $p_{\theta}(\boldsymbol{y} \mid z)$. While one can make use of a purely statistical model by employing e.g. a GP or a (deep) neural network, we advocate here building the surrogate around a coarse-grained model (CGM). The latter can be based on simply coarsening the discretization of the governing equations (Grigo and Koutsourelakis (2019a)) or by employing simplified physics (Grigo and Koutsourelakis $(2019 b))$. It serves as a stencil that automatically retains the primary physical characteristics of the FGM and enables therefore training with small amounts of data.

Let $\boldsymbol{X}$ and $\boldsymbol{Y}$ denote the input and output vector of the aforementioned CGM. The physical meaning of these variables does not need to be the same as $\boldsymbol{x}$ or $\boldsymbol{y}$ but are by construction lowerdimensional and the solution of the CGM i.e. the cost of each evaluation of $\boldsymbol{Y}(\boldsymbol{X})^{2}$ is negligible as compared to $\boldsymbol{y}(\boldsymbol{x})$. We propose:

\footnotetext{
${ }^{2}$ We assume a deterministic CGM for simplicity although this can be relaxed.
} 


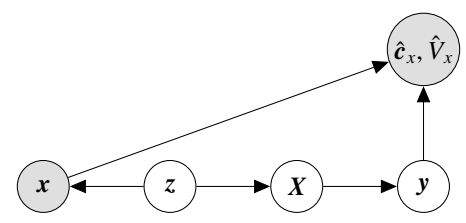

Figure 2: The node $\boldsymbol{X}$ corresponds to the (input) variables of a deterministic coarse-grained model (CGM), implying that $\boldsymbol{z}$ is encouraged not only to learn a representation of the inputs $\boldsymbol{x}$, but in particular features that through the CGM can be predictive of the FGM output $\boldsymbol{y}$ (compare with Figure 1c).

- linking the latent features $z$ with $\boldsymbol{X}$ through a density $p_{\boldsymbol{\theta}}(\boldsymbol{X} \mid \boldsymbol{z})$ with tunable parameters $\boldsymbol{\theta}$

- linking the sought FGM output $\boldsymbol{y}$ with the output of the CGM $\boldsymbol{Y}(\boldsymbol{X})$ rather than with $\boldsymbol{z}$ directly. Hence instead of $p_{\boldsymbol{\theta}}(\boldsymbol{y} \mid z)$ we propose employing a density:

$$
p_{\theta}(\boldsymbol{y} \mid \boldsymbol{Y}(\boldsymbol{X}))
$$

The proposed model implicitly defines an (analytically intractable) conditional density $p_{\boldsymbol{\theta}}(\boldsymbol{y} \mid \boldsymbol{x})$, i.e.:

$$
p_{\theta}(\boldsymbol{y} \mid \boldsymbol{x})=\int p_{\theta}(\boldsymbol{y} \mid \boldsymbol{Y}(\boldsymbol{X})) p_{\theta}(\boldsymbol{X} \mid \boldsymbol{z}) p_{\theta}(z \mid \boldsymbol{x}) \mathrm{d} \boldsymbol{X} \mathrm{d} \boldsymbol{z}
$$

by mapping the the latent encoding $\boldsymbol{z}$ to the effective properties $\boldsymbol{X}$ of a CGM while simultaneously learning to reconstruct the FGM's solution $\boldsymbol{y}(\boldsymbol{x})$ from the output of the CGM by means of $p_{\boldsymbol{\theta}}(\boldsymbol{y} \mid \boldsymbol{Y}(\boldsymbol{X}))$ (Figure 2).

We specify $\boldsymbol{X}, \boldsymbol{Y}$, the CGM itself as well as the densities involved in subsequent sections and in particular in the context of the numerical illustrations (section 3). The introduction of the CGM and the associated latent variables $\boldsymbol{X}$ (and $\boldsymbol{Y}$ for a stochastic CGM) does not alter the generative nature of the model. We note though that the CGM can be omitted or simply complemented by a phenomenological statistical emulator, in which case the graphical model structure in Figure 2 would be altered. 


\subsection{Specification of generative model}

Based on the previous remarks and assuming real-valued $\boldsymbol{x}, \boldsymbol{z}, \boldsymbol{X}, \boldsymbol{y}$ we propose the following probabilistic generative model (for a schematic overview see also Figure 3 )

$$
\begin{array}{rlrl}
\boldsymbol{z} & \sim \mathcal{N}(\mathbf{0}, \boldsymbol{I}) & \\
\boldsymbol{x} & =\boldsymbol{f}\left(z ; \boldsymbol{\theta}_{x}\right)+\boldsymbol{S}_{x}^{1 / 2}\left(z ; \boldsymbol{\theta}_{x}\right) \boldsymbol{\varepsilon}_{x} & \boldsymbol{\varepsilon}_{x} \sim \mathcal{N}(\mathbf{0}, \boldsymbol{I}) \\
\boldsymbol{X} & =\boldsymbol{g}\left(z ; \boldsymbol{\theta}_{g}\right)+\boldsymbol{S}_{X}^{1 / 2} \boldsymbol{\varepsilon}_{X} & \boldsymbol{\varepsilon}_{X} \sim \mathcal{N}(\mathbf{0}, \boldsymbol{I}) \\
\boldsymbol{y} & =\boldsymbol{h}\left(\boldsymbol{Y}(\boldsymbol{X}) ; \boldsymbol{\theta}_{y}\right)+\boldsymbol{S}_{y}^{1 / 2} \boldsymbol{\varepsilon}_{y} & & \boldsymbol{\varepsilon}_{y} \sim \mathcal{N}(\mathbf{0}, \boldsymbol{I})
\end{array}
$$

where $\boldsymbol{f}(\cdot)$ and $\boldsymbol{g}(\cdot)$ are nonlinear functions (e.g. neural networks) parameterized by $\boldsymbol{\theta}_{x}$ and $\boldsymbol{\theta}_{g}$ respectively. We defer discussion of the specifics until section 3 where the meaning of the different variables is presented. In the following we will condition explicitly on $\boldsymbol{\theta}$ in our notation, to avoid ambiguity and to emphasize that $\boldsymbol{\theta}$ (in the most general case) is treated probabilistically as well. Since we operate under the assumption of Small labeled Data, the complexity of $\boldsymbol{g}\left(\boldsymbol{z} ; \boldsymbol{\theta}_{g} \cdot\right)$ is chosen relatively low compared to $\boldsymbol{f}\left(\boldsymbol{z} ; \boldsymbol{\theta}_{x}\right)$, in order to allow learning a mapping from latent space to effective properties $\boldsymbol{X}$ with comparably few examples. The role of $\boldsymbol{h}\left(\boldsymbol{Y}(\boldsymbol{X}) ; \boldsymbol{\theta}_{y}\right)$ is to define the map from the CGM's output $\boldsymbol{Y}(\boldsymbol{X})$ to the (mean of the) output $\boldsymbol{y}$ of the FGM. All the conditional densities in (17) - (19) are multivariate Gaussians which have constant covariances with the exception of Equation (17) where the covariance $\boldsymbol{S}_{x}$ depends on the $\boldsymbol{z}$ variables as dictated by the associated parameters $\boldsymbol{\theta}_{x}$.

We denote by $\boldsymbol{\theta}=\left\{\boldsymbol{\theta}_{x}, \boldsymbol{\theta}_{g}, \boldsymbol{\theta}_{y}, \boldsymbol{S}_{x}, \boldsymbol{S}_{\boldsymbol{X}}, \boldsymbol{S}_{\boldsymbol{y}}\right\}$ the parameters of the generative model, which we wish to learn from a dataset $\mathcal{D}=\left\{\mathcal{D}_{u}, \mathcal{D}_{l}, \mathcal{D}_{O}\right\}$ which, in the most general case, consists of $N_{u}$ unlabeled examples $\mathcal{D}_{u}=\left\{\boldsymbol{x}^{\left(i_{u}\right)}\right\}_{i=1}^{N_{u}}, N_{l}$ labeled input-output examples $\mathcal{D}_{l}=\left\{\left(\boldsymbol{x}^{\left(i_{l}\right)}, \boldsymbol{y}^{\left(i_{l}\right)}\right)\right\}_{i=1}^{N_{l}}$, and tp a collection $\mathcal{D}_{O}=\left\{\boldsymbol{x}^{\left(i_{O}\right)}, \hat{\boldsymbol{o}}^{\left(i_{O}\right)}\right\}_{i_{O}=1}^{N_{O}}$ of query input points and virtual observables. We may then write the marginal likelihood as:

$$
\begin{aligned}
p(\mathcal{D} \mid \boldsymbol{\theta}) & =p\left(\mathcal{D}_{u} \mid \boldsymbol{\theta}\right) p\left(\mathcal{D}_{l} \mid \boldsymbol{\theta}\right) p\left(\mathcal{D}_{O} \mid \boldsymbol{\theta}\right) \\
& =\prod_{i_{u}=1}^{N_{u}} p\left(\boldsymbol{x}^{\left(i_{u}\right)} \mid \boldsymbol{\theta}\right) \prod_{i_{u}=1}^{N_{l}} p\left(\boldsymbol{x}^{\left(i_{l}\right)}, \boldsymbol{y}^{\left(i_{l}\right)} \mid \boldsymbol{\theta}\right) \prod_{i_{O}=1}^{N_{O}} p\left(\boldsymbol{x}^{\left(i_{O}\right)}, \hat{\boldsymbol{o}}^{\left(i_{O}\right)} \mid \boldsymbol{\theta}\right)
\end{aligned}
$$

where each of the likelihood terms in the products are given by Equations (4), (3) and (9) (or (13)) respectively. In view of the densities in Equations (16) - (19) these become:

$$
\begin{aligned}
& p\left(\boldsymbol{x}^{\left(i_{u}\right)} \mid \boldsymbol{\theta}\right)= \int \mathcal{N}\left(\boldsymbol{x}^{\left(i_{u}\right)} \mid \boldsymbol{f}\left(\boldsymbol{z}^{\left(i_{u}\right)} ; \boldsymbol{\theta}_{x}\right), \boldsymbol{S}_{x}\left(\boldsymbol{z}^{\left(i_{u}\right)} ; \theta_{x}\right)\right) \mathcal{N}\left(\boldsymbol{z}^{\left(i_{u}\right)} \mid 0, \boldsymbol{I}\right) d \boldsymbol{z}^{\left(i_{u}\right)}, \\
& p\left(\boldsymbol{x}^{\left(i_{l}\right)}, \boldsymbol{y}^{\left(i_{l}\right)} \mid \boldsymbol{\theta}\right)=\int \mathcal{N}\left(\boldsymbol{y}^{\left(i_{l}\right)} \mid \boldsymbol{h}\left(\boldsymbol{Y}\left(\boldsymbol{X}^{\left(i_{l}\right)}\right) ; \boldsymbol{\theta}_{y}\right), \boldsymbol{S}_{y}\right) \mathcal{N}\left(\boldsymbol{X}^{\left(i_{l}\right)} \mid \boldsymbol{g}\left(\boldsymbol{z}^{\left(i_{l}\right)} ; \boldsymbol{\theta}_{g}\right), \boldsymbol{S}_{X}\right) \\
& \mathcal{N}\left(\boldsymbol{x}^{\left(i_{l}\right)} \mid \boldsymbol{f}\left(\boldsymbol{z}^{\left(i_{l}\right)} ; \boldsymbol{\theta}_{x}\right), \boldsymbol{S}_{x}\left(\boldsymbol{z}^{\left(i_{l}\right)} ; \theta_{x}\right)\right) \mathcal{N}\left(\boldsymbol{z}^{\left(i_{l}\right)} \mid 0, \boldsymbol{I}\right) d \boldsymbol{X}^{\left(i_{l}\right)} d \boldsymbol{z}^{\left(i_{l}\right)}
\end{aligned}
$$

and

$$
\begin{aligned}
p\left(\boldsymbol{x}^{\left(i_{O}\right)}, \hat{\boldsymbol{o}}^{\left(i_{O}\right)} \mid \boldsymbol{\theta}\right)= & \int p\left(\hat{\boldsymbol{o}}^{\left(i_{O}\right)} \mid \boldsymbol{y}^{\left(i_{O}\right)}, \boldsymbol{x}^{\left(i_{O}\right)} ; \boldsymbol{\tau}\right) \mathcal{N}\left(\boldsymbol{y}^{\left(i_{O}\right)} \mid \boldsymbol{h}\left(\boldsymbol{Y}\left(\boldsymbol{X}^{\left(i_{o}\right)}\right) ; \boldsymbol{\theta}_{y}\right), \boldsymbol{S}_{y}\right) \mathcal{N}\left(\boldsymbol{X}^{\left(i_{O}\right)} \mid \boldsymbol{g}\left(\boldsymbol{z}^{\left(i_{O}\right)} ; \boldsymbol{\theta}_{g}\right), \boldsymbol{S}_{X}\right) \\
& \mathcal{N}\left(\boldsymbol{x}^{\left(i_{O}\right)} \mid \boldsymbol{f}\left(\boldsymbol{z}^{\left(i_{l}\right)} ; \boldsymbol{\theta}_{x}\right), \boldsymbol{S}_{x}\left(\boldsymbol{z}^{\left(i_{O}\right)} ; \theta_{x}\right)\right) \mathcal{N}\left(\boldsymbol{z}^{\left(i_{O}\right)} \mid 0, \boldsymbol{I}\right) d \boldsymbol{y}^{\left(i_{O}\right)} d \boldsymbol{X}^{\left(i_{O}\right)} d \boldsymbol{z}^{\left(i_{O}\right)}
\end{aligned}
$$

where $p\left(\hat{\boldsymbol{o}}^{\left(i_{o}\right)} \mid \boldsymbol{y}^{\left(i_{O}\right)}, \boldsymbol{x}^{\left(i_{o}\right)} ; \boldsymbol{\tau}\right)$ depends on the nature of the virtual observable (e.g. Equation (8) or Equation (12)). A fully Bayesian model could be defined by the introduction of appropriate priors for $\boldsymbol{\theta}$ leading to to a posterior on those, i.e. $p(\boldsymbol{\theta} \mid \mathcal{D}) \propto p(\mathcal{D} \mid \boldsymbol{\theta}) p(\boldsymbol{\theta})$. 


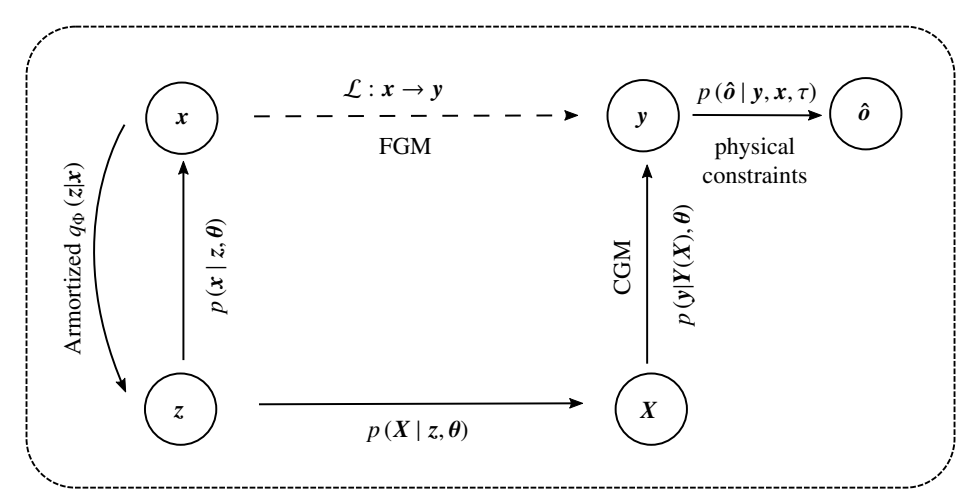

Figure 3: A schematic overview of the constituent parts of the model

\subsection{Inference and Learning}

Our primary objective is to learn the model parameters $\boldsymbol{\theta}$ on the basis of the mixed data $\mathcal{D}=\left\{\mathcal{D}_{u}, \mathcal{D}_{s}, \mathcal{D}_{O}\right\}$ so that the trained probabilistic surrogate can be used for predictive purposes. This task is hindered by the intractability of all the likelihood terms in Equations (22)-(23) due to the presence of the latent variables which must be integrated-out. In particular, we denote summarily by $\mathcal{R}=\left\{\mathcal{Z}_{u}, \mathcal{Z}_{l}, \mathcal{Z}_{O}, \mathfrak{x}_{l}, \mathfrak{x}_{O}, \mathcal{Y}_{O}\right\}$ the latent variables appearing in Equations (21) (23) which consist of:

- $\mathcal{Z}_{u}=\left\{\boldsymbol{z}^{\left(i_{u}\right)}\right\}_{i_{u}=1}^{N_{u}}$ associated with $\mathcal{D}_{u}$ (see e.g. Equation (4) or Equation (21)),

- $\mathcal{Z}_{l}=\left\{z^{\left(i_{l}\right)}\right\}_{i_{l}=1}^{N_{l}}, \mathfrak{X}_{l}=\left\{\boldsymbol{X}^{\left(i_{l}\right)}\right\}_{i_{l}=1}^{N_{l}}$ associated with $\mathcal{D}_{l}$ (see e.g. Equation (3) or (22)),

- $\mathcal{Z}_{O}=\left\{\boldsymbol{z}^{\left(i_{O}\right)}\right\}_{i_{O}=1}^{N_{O}}, \mathfrak{X}_{O}=\left\{\boldsymbol{X}^{\left(i_{O}\right)}\right\}_{i_{O}=1}^{N_{O}}, \boldsymbol{y}_{O}=\left\{\boldsymbol{y}^{\left(i_{O}\right)}\right\}_{i_{O}=1}^{N_{O}}$ associated with $\mathcal{D}_{O}$ (see e.g. Equation (23)).

We advocate the use of Stochastic Variational Inference (SVI, (Paisley et al., 2012; Hoffman et al., 2013)) which produces closed-form approximations of the true posterior $p(\boldsymbol{\theta}, \mathcal{R} \mid \mathcal{D})$ and simultaneously of the model evidence $p(\mathcal{D})$. In contrast to sampling-based procedures (e.g. MCMC, SMC), stochastic variational inference yields biased estimates at the benefit of computational efficiency and computable convergence objectives in the form of the Evidence Lower Bound (ELBO,(Blei et al., 2017)). In particular, we denote the variational approximation to the joint posterior as $q_{\xi}(\boldsymbol{\theta}, \mathcal{R})$ where $\boldsymbol{\xi}$ are its tunable parameters and note that the model evidence $p(\mathcal{D})$ can be lower-bounded as (Bishop, 2006):

$$
\begin{aligned}
\log p(\mathcal{D}) & =\log \int p(\mathcal{D}, \boldsymbol{\theta}, \mathcal{R}) \mathrm{d} \boldsymbol{\theta} \mathrm{d} \mathcal{R} \\
& =\mathcal{F}(\boldsymbol{\xi})+K L\left(q_{\boldsymbol{\xi}}(\boldsymbol{\theta}, \mathcal{R}) \| p(\boldsymbol{\theta}, \mathcal{R} \mid \mathcal{D})\right) \\
& \geq \mathcal{F}(\boldsymbol{\xi})
\end{aligned}
$$

where:

$$
0 \leq K L\left(q_{\xi}(\boldsymbol{\theta}, \mathcal{R}) \| p(\boldsymbol{\theta}, \mathcal{R} \mid \mathcal{D})\right)=-\int q_{\xi}(\boldsymbol{\theta}, \mathcal{R}) \log \left(\frac{p(\boldsymbol{\theta}, \mathcal{R} \mid \mathcal{D})}{q_{\xi}(\boldsymbol{\theta}, \mathcal{R})}\right) d \boldsymbol{\theta} d \mathcal{R}
$$


is the $\mathrm{KL}$-divergence between approximate and true posterior, and $\mathcal{F}(\boldsymbol{\xi})$ is the ELBO i.e.

$$
\begin{aligned}
\mathcal{F}(\xi) & =\int q_{\xi}(\boldsymbol{\theta}, \mathcal{R}) \log \frac{p(\mathcal{D}, \boldsymbol{\theta}, \mathcal{R})}{q_{\xi}(\boldsymbol{\theta}, \mathcal{R})} d \boldsymbol{\theta} d \mathcal{R} \\
& =\mathbb{E}_{q_{\xi}}\left[\log \frac{p(\mathcal{D}, \boldsymbol{\theta}, \mathcal{R})}{q_{\xi}(\boldsymbol{\theta}, \mathcal{R})}\right]
\end{aligned}
$$

Maximizing the ELBO over the parameters $\boldsymbol{\xi}$ is therefore equivalent to minimizing the KLdivergence from the true posterior. The ELBO provides a score function for comparing different approximations (e.g. different family of distributions $q \in Q$ or different parametrizations $\boldsymbol{\xi}$ ) and as an approximation to the model evidence can also be used to compare different models (e.g. with different structure or different parametrizations $\boldsymbol{\theta}$ ).

We employ a (partial) mean field approximation i.e. a $q_{\xi}$ that factorizes as follows:

$$
q_{\xi}(\boldsymbol{\theta}, \mathcal{R})=q_{\xi}(\boldsymbol{\theta}) \prod_{i_{u}=1}^{N_{u}} q_{\xi}\left(\boldsymbol{z}^{\left(i_{u}\right)}\right) \prod_{i_{l}=1}^{N_{l}} q_{\xi}\left(\boldsymbol{z}^{\left(i_{i}\right)}\right) q_{\xi}\left(\boldsymbol{X}^{\left(i_{i}\right)}\right) \prod_{i_{o}}^{N_{o}} q_{\xi}\left(z^{(i o}\right) q_{\xi}\left(\boldsymbol{X}^{\left(i_{o}\right)}\right) q_{\xi}\left(\boldsymbol{y}^{\left(i_{o}\right)}\right) .
$$

While this might appear drastic, we note that the elements of $\mathcal{Z}_{u}$ are conditionally (given $\boldsymbol{\theta}$ ) independent of the rest even in the true posterior, as also the latent variables in the following two groups $\left\{\mathcal{Z}_{l}, \mathfrak{F}_{l}\right\}$ and $\left\{\mathcal{Z}_{O}, \mathfrak{X}_{O}, \mathcal{Y}_{O}\right\}$. Given this, the ELBO becomes:

$$
\begin{aligned}
& \mathcal{F}(\boldsymbol{\xi})=\mathbb{E}_{q_{\xi}}\left[\log \left(\frac{p(\mathcal{D}, \boldsymbol{\theta}, \mathcal{R})}{q_{\boldsymbol{\xi}}(\boldsymbol{\theta}, \mathcal{R})}\right)\right] \\
& =\mathbb{E}_{q_{\xi}}\left[\log p\left(\mathcal{D}_{u} \mid \boldsymbol{\theta}, \mathcal{R}\right)+\log p\left(\mathcal{D}_{l} \mid \boldsymbol{\theta}, \mathcal{R}\right)+\log p\left(\mathcal{D}_{O} \mid \boldsymbol{\theta}, \mathcal{R}\right)+\log p(\mathcal{R}, \boldsymbol{\theta})-\log q_{\boldsymbol{\xi}}(\boldsymbol{\theta}, \mathcal{R})\right] \\
& =\sum_{i_{u}=1}^{N_{u}} \mathbb{E}_{q_{\xi}}\left[\log p\left(\boldsymbol{x}^{\left(i_{u}\right)} \mid z^{\left(i_{u}\right)}, \boldsymbol{\theta}\right)\right] \\
& +\sum_{i_{l}=1}^{N_{l}} \mathbb{E}_{q_{\xi}}\left[\log p\left(\boldsymbol{y}^{\left(i_{l}\right)} \mid \boldsymbol{X}^{\left(i_{l}\right)}, \boldsymbol{\theta}\right)+\log p\left(\boldsymbol{x}^{\left(i_{l}\right)} \mid z^{\left(i_{l}\right)}, \boldsymbol{\theta}\right)\right] \\
& \mathbb{E}_{q_{\xi}}\left[\log p\left(\mathcal{D}_{u} \mid \boldsymbol{\theta}, \mathcal{R}\right)\right] \\
& +\sum_{i_{O}=1}^{N_{O}} \mathbb{E}_{q_{\xi}}\left[\log p\left(\hat{\boldsymbol{\sigma}}^{\left(i_{o}\right)} \mid \boldsymbol{y}^{\left(i_{O}\right)}, \boldsymbol{x}^{\left(i_{O}\right)}, \boldsymbol{\theta}\right)+\log p\left(\boldsymbol{x}^{\left(i_{O}\right)} \mid \boldsymbol{z}^{\left(i_{O}\right)}, \boldsymbol{\theta}\right)\right] \quad \mathbb{E}_{q_{\xi}}\left[\log p\left(\mathcal{D}_{O} \mid \boldsymbol{\theta}, \mathcal{R}\right)\right] \\
& +\sum_{i_{u}=1}^{N_{u}} \mathbb{E}_{q_{\xi}}\left[\log p\left(z^{\left(i_{u}\right)}\right)\right] \\
& +\sum_{i_{l}=1}^{N_{l}} \mathbb{E}_{q_{\xi}}\left[\log p\left(\boldsymbol{X}^{\left(i_{l}\right)} \mid z^{\left(i_{l}\right)}, \boldsymbol{\theta}\right)+\log p\left(z^{(i)}\right)\right] \\
& +\sum_{i_{O}=1}^{N_{O}} \mathbb{E}_{q_{\xi}}\left[\log p\left(\boldsymbol{y}^{\left(i_{O}\right)} \mid \boldsymbol{X}^{\left(i_{O}\right)}, \boldsymbol{\theta}\right)+\log p\left(\boldsymbol{X}^{\left(i_{O}\right)} \mid z^{\left(i_{O}\right)}, \boldsymbol{\theta}\right)+\log p\left(\boldsymbol{z}^{\left(i_{O}\right)}\right)\right] \\
& +\mathbb{E}_{q_{\xi}}[\log p(\boldsymbol{\theta})] \\
& -\mathbb{E}_{q_{\xi}}\left[\log q_{\xi}(\mathcal{R})+\log q_{\xi}(\boldsymbol{\theta})\right] .
\end{aligned}
$$

In all subsequent illustrations we used point estimates for the parameters $\theta$ i.e. computed their maximum-a-posteriori (MAP) estimate $\boldsymbol{\theta}_{M A P}$. This is equivalent to introducing a Dirac-delta:

$$
q_{\xi}(\boldsymbol{\theta})=\delta\left(\boldsymbol{\theta}-\boldsymbol{\theta}_{M A P}\right)
$$


in the variational approximation in which case the parameters $\boldsymbol{\xi}$ include also $\boldsymbol{\theta}_{M A P}$. In this case, the expectations with respect to $q_{\xi}(\boldsymbol{\theta})$ can simply be computed by substituting $\boldsymbol{\theta}_{M A P}$ wherever $\boldsymbol{\theta}$ appears and the entropy term $\mathbb{E}_{q_{\xi}}\left[\log q_{\xi}(\boldsymbol{\theta})\right]$ can be ignored as it is independent of $\theta_{M A P}$.

The presence of three sets of conditionally independent datasets i.e. $\mathcal{D}_{u}, \mathcal{D}_{l}$ and $\mathcal{D}_{O}$ (Equation (20)) leads to an additive decomposition of the ELBO of the form $\mathcal{F}=\mathcal{F}_{u}+\mathcal{F}_{l}+\mathcal{F}_{O}+\log p\left(\boldsymbol{\theta}_{M A P}\right)$, where

$$
\mathcal{F}_{u}(\boldsymbol{\xi})=\sum_{i_{u}=1}^{N_{u}} \mathbb{E}_{q_{\xi}}\left[p\left(\boldsymbol{x}^{\left(i_{u}\right)} \mid \boldsymbol{z}^{\left(i_{u}\right)}, \boldsymbol{\theta}\right)\right]+\sum_{i_{u}=1}^{N_{u}} \mathbb{E}_{q_{\xi}}\left[\log p\left(\boldsymbol{z}^{\left(i_{u}\right)}\right)\right]-\sum_{i_{u}=1}^{N_{u}} \mathbb{E}_{q_{\xi}}\left[\log q_{\xi}\left(\boldsymbol{z}^{\left(i_{u}\right)}\right)\right]
$$

accounts for the terms associated with the unlabeled data $\mathcal{D}_{u}$,

$$
\begin{aligned}
\mathcal{F}_{l}(\boldsymbol{\xi}) & =\sum_{i_{l}=1}^{N_{l}} \mathbb{E}_{q_{\xi}}\left[\log p\left(\boldsymbol{y}^{\left(i_{l}\right)} \mid \boldsymbol{X}^{\left(i_{l}\right)}, \boldsymbol{\theta}\right)+\log p\left(\boldsymbol{x}^{\left(i_{l}\right)} \mid \boldsymbol{z}^{\left(i_{l}\right)}, \boldsymbol{\theta}\right)\right] \\
& +\sum_{i_{l}=1}^{N_{l}} \mathbb{E}_{q_{\xi}}\left[\log p\left(\boldsymbol{X}^{\left(i_{l}\right)} \mid \boldsymbol{z}^{\left(i_{l}\right)}, \boldsymbol{\theta}\right)+\log p\left(\boldsymbol{z}^{\left(i_{l}\right)}\right)\right] \\
& -\sum_{i_{l}=1}^{N_{l}} \mathbb{E}_{q_{\xi}}\left[\log q_{\xi}\left(\boldsymbol{X}^{\left(i_{l}\right)}\right)+\log q_{\xi}\left(\boldsymbol{z}^{\left(i_{l}\right)}\right)\right]
\end{aligned}
$$

accounts for the terms associated with the labeled data $\mathcal{D}_{l}$, and

$$
\begin{aligned}
\mathcal{F}_{O}(\boldsymbol{\xi}) & =\sum_{i_{O}=1}^{N_{O}} \mathbb{E}_{q_{\xi}}\left[\log p\left(\hat{\boldsymbol{o}}^{\left(i_{O}\right)} \mid \boldsymbol{y}^{\left(i_{O}\right)}, \boldsymbol{x}^{\left(i_{O}\right)}, \boldsymbol{\theta}\right)+\log p\left(\boldsymbol{x}^{\left(i_{O}\right)} \mid \boldsymbol{z}^{\left(i_{O}\right)}, \boldsymbol{\theta}\right)\right] \\
& +\sum_{i_{O}=1}^{N_{O}} \mathbb{E}_{q_{\xi}}\left[\log p\left(\boldsymbol{y}^{\left(i_{o}\right)} \mid \boldsymbol{X}^{\left(i_{O}\right)}, \boldsymbol{\theta}\right)+\log p\left(\boldsymbol{X}^{\left(i_{o}\right)} \mid \boldsymbol{z}^{\left(i_{O}\right)}, \boldsymbol{\theta}\right)+\log p\left(\boldsymbol{z}^{\left(i_{O}\right)}\right)\right] \\
& -\sum_{i_{l}=1}^{N_{O}} \mathbb{E}_{q_{\xi}}\left[\log q_{\xi}\left(\boldsymbol{y}^{\left(i_{l}\right)}\right)+\log q_{\boldsymbol{\xi}}\left(\boldsymbol{X}^{\left(i_{l}\right)}\right)+\log q_{\xi}\left(\boldsymbol{z}^{\left(i_{l}\right)}\right)\right]
\end{aligned}
$$

accounts for the terms associated with the virtual observables/data $\mathcal{D}_{O}$.

We note that in Equation (30), Equation (31) and Equation (32) the expected log-likelihood terms (i.e. first sum) promote a good fit of the generative model to the unlabeled $\mathcal{D}_{u}$, labeled $\mathcal{D}_{l}$ and virtual data $\mathcal{D}_{v o}$ data respectively, while the second and third sums correspond to the Kullback-Leibler divergence between approximate posteriors and priors which act as regularization that prevents overfitting. The common model parameters $\boldsymbol{\theta}$ appear in all components of the ELBO and synthesize the information provided by the different data-types. We highlight the term $\log p\left(\hat{\boldsymbol{o}}^{\left(i_{O}\right)} \mid \boldsymbol{y}^{\left(i_{O}\right)}, \boldsymbol{x}^{\left(i_{O}\right)}, \boldsymbol{\theta}\right)$ in Equation (32), which is is driven by the virtual dataset and reflects the incorporation of our (in)equality constraints. In this case, the model attempts to infer the solution $\boldsymbol{y}^{\left(i_{O}\right)}$ through $q_{\xi}\left(\boldsymbol{y}^{\left(i_{O}\right)}\right)$. Hence the updates of the model parameters $\boldsymbol{\theta}$ are affected also by the inferred solutions and the uncertainty associated with them.

For the structured mean-field approximation $q_{\xi}(\boldsymbol{\theta}, \mathcal{R})$ in Equation (27) we adopt diagonal Gaussians, primarily due to their linear scaling with the dimension of the corresponding latent variables. The following forms and parametrizations for the variational posteriors $q_{\xi}$ in Equation (27) were adopted:

- $\forall i_{u} \in\left\{1, \ldots, N_{u}\right\}: \quad q_{\xi}\left(z^{\left(i_{u}\right)}\right)=\mathcal{N}\left(z^{\left(i_{l}\right)} \mid \boldsymbol{\mu}_{z}^{\left(i_{u}\right)}, \operatorname{diag}\left(\sigma_{z}^{\left(i_{u}\right)}\right)\right)$

- $\forall i_{l} \in\left\{1, \ldots, N_{l}\right\}: \quad q_{\xi}\left(z^{\left(i_{i}\right)}\right)=\mathcal{N}\left(z^{\left(i_{l}\right)} \mid \boldsymbol{\mu}_{z}^{\left(i_{l}\right)}, \operatorname{diag}\left(\boldsymbol{\sigma}_{z}^{\left(i_{l}\right)}\right)\right) \quad q_{\boldsymbol{\xi}}\left(\boldsymbol{X}^{\left(i_{l}\right)}\right)=\mathcal{N}\left(\boldsymbol{X}^{\left(i_{l}\right)} \mid \boldsymbol{\mu}_{\boldsymbol{X}}^{\left(i_{l}\right)}, \operatorname{diag}\left(\boldsymbol{\sigma}_{\boldsymbol{X}}^{\left(i_{l}\right)}\right)\right)$

- $\forall i_{O} \in\left\{1, \ldots, N_{O}\right\}: \quad q_{\xi}\left(z^{\left(i_{O}\right)}\right)=\mathcal{N}\left(z^{\left(i_{O}\right)} \mid \boldsymbol{\mu}_{z}^{\left(i_{O}\right)}, \operatorname{diag}\left(\boldsymbol{\sigma}_{z}^{\left(i_{O}\right)}\right)\right) \quad q_{\xi}\left(\boldsymbol{X}^{\left(i_{O}\right)}\right)=\mathcal{N}\left(\boldsymbol{X}^{\left(i_{O}\right)} \mid \boldsymbol{\mu}_{\boldsymbol{X}}^{\left(i_{O}\right)}, \operatorname{diag}\left(\boldsymbol{\sigma}_{\boldsymbol{X}}^{\left(i_{O}\right)}\right)\right)$

$$
q_{\xi}\left(\boldsymbol{y}^{\left(i_{O}\right)}\right)=\mathcal{N}\left(\boldsymbol{y}^{\left(i_{O}\right)} \mid \boldsymbol{\mu}_{\boldsymbol{y}}^{\left(i_{O}\right)}, \operatorname{diag}\left(\boldsymbol{\sigma}_{\boldsymbol{y}}^{\left(i_{O}\right)}\right)\right)
$$


which, in combination with Equation (29) suggest that the parameter vector $\xi$ consists of:

$$
\boldsymbol{\xi}=\left\{\boldsymbol{\theta}_{M A P},\left\{\boldsymbol{\mu}_{z}^{\left(i_{u}\right)}, \boldsymbol{\sigma}_{z}^{\left(i_{u}\right)}\right\}_{i=1}^{N_{u}}\left\{\mu_{z}^{\left(i_{l}\right)}, \sigma_{z}^{\left(i_{l}\right)}, \mu_{\boldsymbol{X}}^{\left(i_{l}\right)}, \sigma_{\boldsymbol{X}}^{\left(i_{l}\right)}\right\}_{i=1}^{N_{l}}\left\{\boldsymbol{\mu}_{z}^{\left(i_{O}\right)}, \boldsymbol{\sigma}_{z}^{\left(i_{O}\right)}, \boldsymbol{\mu}_{\boldsymbol{X}}^{\left(i_{O}\right)}, \boldsymbol{\sigma}_{\boldsymbol{X}}^{\left(i_{O}\right)}, \boldsymbol{\mu}_{\boldsymbol{y}}^{\left(i_{O}\right)}, \boldsymbol{\sigma}_{\boldsymbol{y}}^{\left(i_{O}\right)}\right\}_{i=1}^{N_{O}}\right\}
$$

For the parameters that are constrained to be positive, a suitable transformation (e.g. $\exp (\cdot))$ is employed such that maximizing the ELBO becomes an unconstrained optimization problem ${ }^{3}$.

We note further that, since we target cases where that the number of labeled data points $N_{l}$ is as small as possible due to the numerical cost of the forward problem, one could potentially leverage the larger number of $N_{u}$ unlabeled data which carry negligible cost. From Equation (33) it is obvious that the number of variational parameters with $\mathcal{D}_{u}$ scales linearly with $N_{u}$. One may therefore consider introducing an amortized encoder $q_{\Phi}\left(z^{\left(i_{u}\right)} \mid \boldsymbol{x}^{\left(i_{u}\right)}\right)$ (Kingma and Welling, 2013), i.e. an approximate posterior that explicitly accounts for the dependence of each $z^{\left(i_{u}\right)}$ on the data $\boldsymbol{x}^{\left(i_{u}\right)}$. In particular, we adopt an approximate posterior of the form:

$$
q_{\Phi}\left(z^{\left(i_{u}\right)} \mid \boldsymbol{x}^{\left(i_{u}\right)}\right)=\mathcal{N}\left(\boldsymbol{z}^{\left(i_{u}\right)} \mid \boldsymbol{\mu}_{\Phi}\left(\boldsymbol{x}^{\left(i_{u}\right)}\right), \operatorname{diag}\left(\boldsymbol{\sigma}_{\Phi}\left(\boldsymbol{x}^{\left(i_{u}\right)}\right)\right)\right) \quad \forall i \in\left\{1, \ldots, N_{u}\right\}
$$

where the parameters $\Phi$ are the same for all $i_{u}$. While the approximate posterior in Equation (34) can, at best, achieve the same ELBO as the $q_{\xi}\left(z^{\left(i_{u}\right)}\right)$ above, it contains fewer parameters that need to be optimized (at least for large $N_{u}$ ) and once trained can be readily used as an approximation to the true posterior $p_{\theta}(z \mid x)$ for predictive purposes in Equation (15). In our simulations, the parameters $\Phi$ pertain to deep neural nets (see section 3) and from a practical point of view, the only difference is that $\left\{\boldsymbol{\mu}_{z}^{\left(i_{u}\right)}, \sigma_{z}^{\left(i_{u}\right)}\right\}_{i=1}^{N_{u}}$ are substituted by the parameters $\Phi$ in the vector $\boldsymbol{\xi}$ of Equation (33), and that the unlabeled data is subsampled in batches during training.

We conclude this section by enumerating the basic steps associated with the variational inference task in Algorithm 1. The intractable expectations with respect to $q_{\xi}$ appearing in the ELBO $\mathcal{F}$ and its gradient $\nabla_{\xi} \mathcal{F}$ are estimated with Monte Carlo. In order to reduce the variance of these estimators, we apply the well-established reparametrization trick (Kingma and Welling, 2013).

We combine the noisy estimates of the gradient $\nabla_{\xi} \mathcal{F}$ with stochastic gradient ascent (Robbins and Monro, 1951) and the Adam algorithm in particular (Kingma and Ba, 2014). We note that training requires the propagation of gradients through the whole model, including the CGM and the constraints associated with virtual observables. Propagating gradients through the model can readily be done using algorithmic differentiation (Naumann, 2012) whenever possible; i.e. when evaluating a Monte Carlo estimate of the evidence lower bound $\mathcal{F}$ a computational graph is built, such that in a backward pass gradient information propagates from $\mathcal{F}$ to the leaf nodes of the computational graph (e.g. given by the variational parameters $\boldsymbol{\xi}$ ) (Paszke et al., 2017). The CGM and the virtual observables $\boldsymbol{o}(\boldsymbol{y} ; \boldsymbol{x})$ must be embedded within this computational graph, i.e require that the CGM also allows the back-propagation of gradient information. If the CGM is also given by differential equation, the reverse-flow of information required during back-propagation corresponds to the solution of the adjoint problem, at a cost equivalent to the forward solution of the CGM. Obtaining derivatives of the virtual observables is equally a cheap operation but also problem-specific and discussion is deferred until section 3.3.

\footnotetext{
${ }^{3}$ We note that $\sigma$ denotes a vector of variances, not standard deviations.
} 


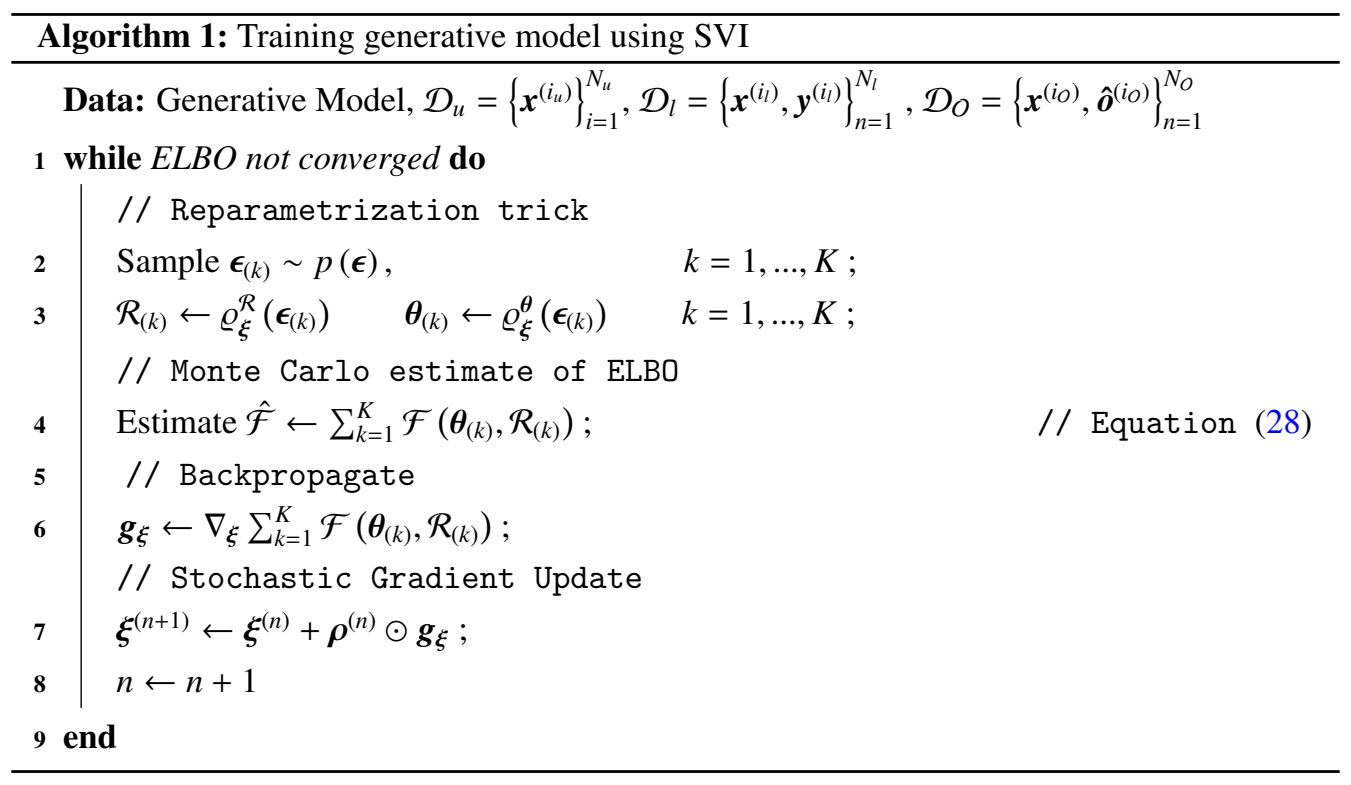

\subsection{Predictions}

Given the (approximate) posterior $q_{\xi}(\boldsymbol{\theta})$ on the model parameters $\boldsymbol{\theta}$ obtained after training, the model can be employed for predictive purposes. In the simplest case, given a new (unobserved) input $\boldsymbol{x}$, we seek the corresponding output $\boldsymbol{y}$. The probabilistic nature of the proposed generative model yields a probability density on $\boldsymbol{y}$ (see also Equation (5)) i.e. the predictive posterior $p(\boldsymbol{y} \mid \boldsymbol{x}, \mathcal{D})$ given by

$$
\begin{aligned}
p(\boldsymbol{y} \mid \boldsymbol{x}, \mathcal{D}) & =\int p(\boldsymbol{y} \mid \boldsymbol{X}, \boldsymbol{\theta}) p(\boldsymbol{X} \mid \boldsymbol{z}, \boldsymbol{\theta}) p(\boldsymbol{z} \mid \boldsymbol{x}, \boldsymbol{\theta}) p(\boldsymbol{\theta} \mid \mathcal{D}) \mathrm{d} z \mathrm{~d} \boldsymbol{X} \mathrm{d} \boldsymbol{\theta} \\
& \approx \int p\left(\tilde{\boldsymbol{y}} \mid \boldsymbol{X}, \boldsymbol{\theta}_{M A P}\right) p\left(\boldsymbol{X} \mid \boldsymbol{z}, \boldsymbol{\theta}_{M A P}\right) p\left(\boldsymbol{z} \mid \boldsymbol{x}, \boldsymbol{\theta}_{M A P}\right) \mathrm{d} \boldsymbol{X} \mathrm{d} \boldsymbol{z}
\end{aligned}
$$

where the variational approximation $q_{\xi}(\boldsymbol{\theta})=\delta\left(\boldsymbol{\theta}-\theta_{M A P}\right)$ was used in place of the intractable posterior $p(\boldsymbol{\theta} \mid \mathcal{D})$. We also briefly mention the possibility (without pursuing it further in this work) to incorporate (additional) constraints $\boldsymbol{o}(\boldsymbol{y} ; \boldsymbol{x})$ at $\boldsymbol{x}$ during the prediction stage as well, i.e. to perform prediction by inference and update the posterior predictive using again the virtual likelihood

$$
p(\boldsymbol{y} \mid \boldsymbol{x}, \hat{\boldsymbol{o}}, \mathcal{D}) \propto p(\hat{\boldsymbol{o}} \mid \boldsymbol{y}, \boldsymbol{x}) p(\boldsymbol{y} \mid \boldsymbol{x}, \mathcal{D})
$$

where $\hat{\boldsymbol{o}}$ denotes the associated virtual observables.

If an amortized approximate posterior $q_{\Phi}(z \mid x)$ has been found in the inference step as detailed in the previous section, then this can be used in place of $p\left(z \mid x, \boldsymbol{\theta}_{M A P}\right)$ in Equation (36). Alternatively, one might employ sampling methods e.g. MCMC or another round of (stochastic) variational inference in order to obtain an approximation, say $q_{\zeta}(z)$. The latter is found by maximizing an analogous ELBO, i.e.: 


$$
\begin{aligned}
q^{*}(z) & =\arg \min _{\zeta} \operatorname{KL}\left[q_{\zeta}(z) \| p\left(z \mid x, \boldsymbol{\theta}_{M A P}\right)\right] \\
& =\arg \max _{\zeta} \mathbb{E}_{q_{\zeta}(z)}\left[\log p\left(\boldsymbol{x} \mid z, \boldsymbol{\theta}_{M A P}\right)\right]-\operatorname{KL}\left[q_{\zeta}(z) \| p(z)\right] \\
& =\arg \max _{\zeta} \hat{\mathcal{F}}_{u}\left(q_{\zeta}(z)\right)
\end{aligned}
$$

We note that in neither of the latter two cases, any additional model solves are required and in the results reported in subsequent sections the variational approximation $q_{\zeta}$ was used. The integral in the predictive posterior of (36) can be approximated with Monte Carlo and requires solely solutions of the CGM. In Algorithm 2 we briefly summarize how probabilistic predictions $p(\boldsymbol{y} \mid \boldsymbol{x}, \mathcal{D})$ can be obtained for new (unobserved) inputs $\boldsymbol{x}$.

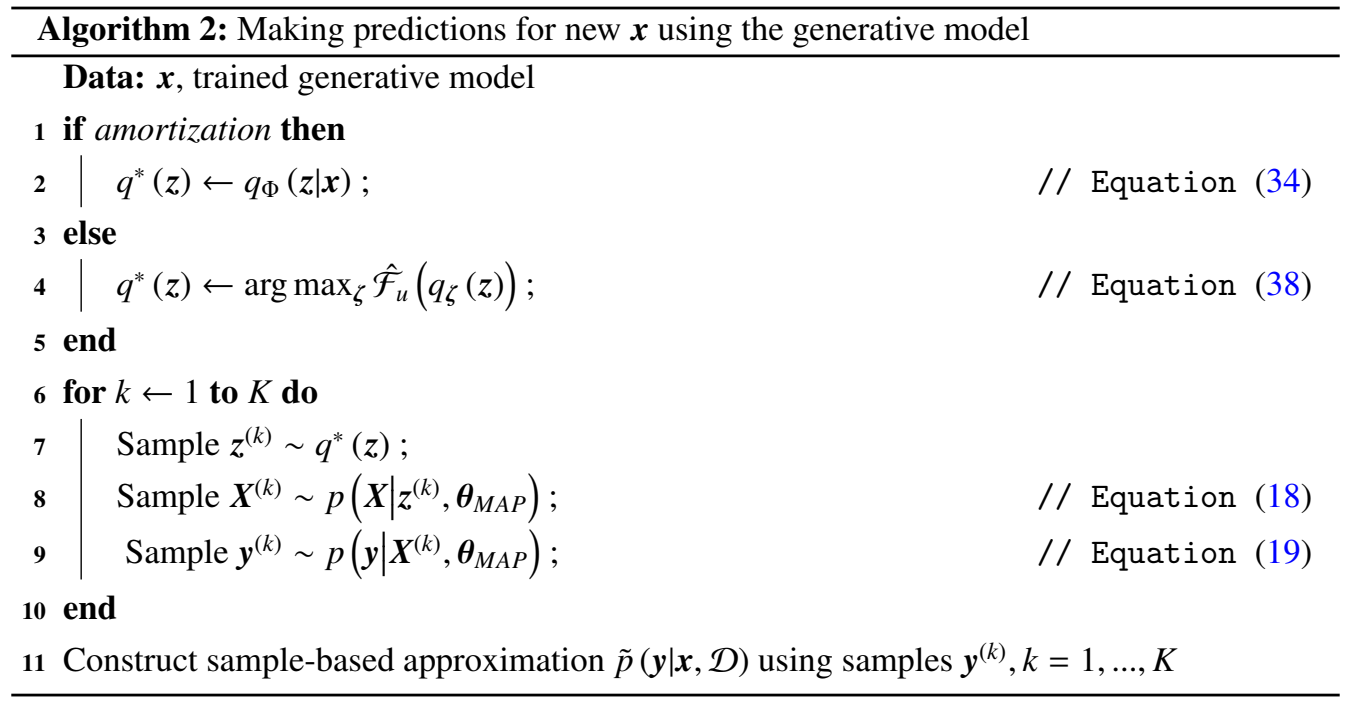

\subsubsection{Predictive performance metrics}

For the purpose of quantifying the predictive performance, we consider a validation dataset $\mathcal{D}_{v}=\left\{\boldsymbol{x}^{\left(i_{v}\right)}, \boldsymbol{y}^{\left(i_{v}\right)}\right\}_{i_{v}=1}^{N_{v}}$ consisting of $N_{v}$ input-output pairs of the FGM, for which we evaluate the following two metrics using the posterior predictive density:

Coefficient of determination $R^{2}$ The coefficient of determination $R^{2}$ is a standard metric (Zhang, 2017) which assesses the accuracy of point estimates, and in particular of the mean $\boldsymbol{\mu}\left(\boldsymbol{x}^{\left(i_{v}\right)}\right)$ of the predictive posterior of our trained model for each validation input $\boldsymbol{x}^{\left(i_{v}\right)}$ i.e.:

$$
\boldsymbol{\mu}\left(\boldsymbol{x}^{\left(i_{v}\right)}\right)=\mathbb{E}_{p\left(y \mid x^{(i v)}, \mathcal{D}\right)}[\boldsymbol{y}], \quad i_{v}=1, \ldots, N_{v} .
$$

The mean of the posterior predictive is estimated using Monte Carlo (see Algorithm 2) and 
is compared to the reference FGM outputs $\left\{\boldsymbol{y}^{\left(i_{v}\right)}\right\}_{i=1}^{N_{\text {val }}}$ as follows:

$$
R^{2}=1-\frac{\sum_{i_{v}=1}^{N_{v}}\left\|\boldsymbol{y}^{\left(i_{v}\right)}-\boldsymbol{\mu}\left(\boldsymbol{x}^{\left(i_{v}\right)}\right)\right\|_{2}^{2}}{\sum_{i_{v}=1}^{N_{v}}\left\|\boldsymbol{y}^{\left(i_{v}\right)}-\boldsymbol{y}_{v}\right\|_{2}^{2}}
$$

where $\boldsymbol{y}_{v}=\frac{1}{N_{v}} \sum_{i_{v}=1}^{N_{v}} \boldsymbol{y}^{\left(i_{v}\right)}$ is the sample average of the validation dataset. It can be noted that $R^{2}$ attains its maximum value, i.e. $R^{2}=1$, when the mean predictive estimates coincide with the actual FGM outputs in the validation dataset and deviations from these are weighted by the variability of the validation data appearing in the denominator of Equation (40).

Logscore $L S$ This metric assess not just point estimates of the predictive posterior but also the associated predictive uncertainty. In particular and for the purpose of computing $L S$ we approximate the otherwise intractable $p\left(\boldsymbol{y} \mid \boldsymbol{x}^{\left(i_{v}\right)}, \mathcal{D}\right)$ in Equation (36) at each validation input $\boldsymbol{x}^{\left(i_{v}\right)}$, by a Gaussian with a mean equal to the actual mean of the predictive posterior $\boldsymbol{\mu}\left(\boldsymbol{x}^{\left(i_{v}\right)}\right)$ (Equation (39) - estimated by Monte Carlo) and a diagonal covariance matrix $\boldsymbol{S}\left(\boldsymbol{x}^{\left(i_{v}\right)}\right)$ containing the actual variances (also estimated by Monte Carlo - see Algorithm 2), i.e.:

$$
\boldsymbol{S}\left(\boldsymbol{x}^{\left(i_{v}\right)}\right)=\operatorname{diag}\left(\sigma_{j}^{2}\left(\boldsymbol{x}^{\left(i_{v}\right)}\right)\right), \quad i_{v}=1, \ldots, N_{v}
$$

where

$$
\sigma_{j}^{2}\left(\boldsymbol{x}^{\left(i_{v}\right)}\right)=\mathbb{E}_{p\left(y \mid x^{\left(i_{v}\right)}, \mathcal{D}\right)}\left[\left(y_{j}-\mu_{j}\left(\boldsymbol{x}^{\left(i_{v}\right)}\right)\right)^{2}\right], \quad i_{v}=1, \ldots, N_{v} .
$$

Subsequently, $L S$ is evaluated as follows:

$$
L S=\frac{1}{N_{v}} \sum_{i_{v}=1}^{N_{v}} \log \mathcal{N}\left(\boldsymbol{y}^{\left(i_{v}\right)} \mid \boldsymbol{\mu}\left(\boldsymbol{x}^{\left(i_{v}\right)}\right), \boldsymbol{S}\left(\boldsymbol{x}^{\left(i_{v}\right)}\right)\right) .
$$

One notes that high $L S$ values are achieved not only when the predictive mean $\boldsymbol{\mu}\left(\boldsymbol{x}^{\left(i_{t}\right)}\right)$ is close to the true $\boldsymbol{y}^{\left(i_{t}\right)}$ but also when the predictive uncertainty (as measured by the variances $\sigma_{j}^{2}\left(\boldsymbol{x}^{\left(i_{t}\right)}\right)$ ) is simultaneously as small as possible. It can finally be shown (Grigo and Koutsourelakis, 2019a) that $L S$ approximates the Kullback-Leibler divergence between the true $\pi(\boldsymbol{y} \mid \boldsymbol{x})$ and the (Gaussian approximation of the) predictive posterior $p(\boldsymbol{y} \mid \boldsymbol{x}, \mathcal{D})$ averaged over the true distribution, say $\pi(\boldsymbol{x})$, of the inputs.

\section{Numerical Illustrations}

We demonstrate the capabilities of the proposed framework in discovering predictive, probabilistic surrogates on a two-dimensional diffusion problem. In the sequel, we specify particular elements of the proposed model that were presented generically in the previous sections and concretize parametrizations and their meaning. The goals of the numerical illustrations are:

- to examine the effect of the number labeled data $N_{l}$ which are the most expensive to obtain and to assess whether the model can perform well under small $N_{l}$ (i.e. a few tens of FGM runs, section 3.4 ). 
- to assess the ability of the model to learn effective and interpretable CGMs that provide insight to the relevant features of the high-dimensional input $\boldsymbol{x}$ which are predictive of the output $\boldsymbol{y}$ (section 3.4).

- to examine the effect of the amount of virtual observables $\mathcal{D}_{O}$ and assess whether the model's predictive performance can be improved by increasing the number $N_{O}$ of such data (section 3.5).

- to examine the effect of the type of virtual observables provided for training. In particular, we consider three different types (namely coarse-grained residuals, hybrid and potential energy) and assess the model's predictive performance for each one of those (section 3.5).

- to examine the effect of unlabeled data $\mathcal{D}_{u}$ which are inexpensive to obtain and assess whether the model's predictive performance can be improved by increasing the number $N_{u}$ of such data (section 3.6).

- to examine the effect of the information bottleneck implied by the latent variables $z$ and the CGM and to assess the effect of the the dimension of $z$ and the CGM's state variables (i.e. $\boldsymbol{X}$ and $\boldsymbol{Y}$ ) on the predictive performance of the model (section 3.7)

- to assess the predictive performance of the model under high-dimensional parametric inputs $\boldsymbol{x}$ and under "interpolative" and "extrapolative" conditions. The latter distinction refers to the ability to predict the (equally high-dimensional) output vector $\boldsymbol{y}$ under boundary conditions that were used during training (interpolative) or not (extrapolative) (section $3.8)$.

- to investigate the efficiency and accuracy of the trained surrogate in a many-query application involving uncertainty propagation (section 3.9 ).

Some of the simulation results as well as the corresponding code will be made available at the following github repository ${ }^{4}$ upon publication.

${ }^{4}$ https://github.com/bdevl/PGMCPC 


\subsection{Definition of physical problem}

For the numerical illustration of our modeling framework we consider a linear elliptic PDE defined on the unit square $\Omega=[0,1]^{d}$ in dimension $d=2$. We can write the governing equations as a two-field problem

$$
\begin{gathered}
\text { conservation law: } \nabla \cdot \boldsymbol{J}(\boldsymbol{s})=f, \quad \forall s \in \Omega \\
\text { constitutive law: } \boldsymbol{J}(\boldsymbol{s})=-\nabla(\kappa(s) u(s)) \quad \forall s \in \Omega
\end{gathered}
$$

with boundary conditions:

$$
\begin{gathered}
u=u_{D}, \quad s \in \Gamma_{D} \\
\boldsymbol{J} \cdot \boldsymbol{n}=\mathbf{0}, \quad \boldsymbol{s} \in \Gamma_{N}
\end{gathered}
$$

where $u(s)$ is a scalar field to which one might attribute the physical meaning of temperature or pressure or concentration, $\boldsymbol{J}(\boldsymbol{s})$ is a vector field representing flux, and $\boldsymbol{n}$ is the unit outward normal vector. $\Gamma_{N}$ denotes the part of the boundary where Neumann boundary conditions are prescribed and is comprised of the top and bottom sides of the unit square $\Omega$ i.e. for $\left\{s \mid s_{2}=0\right.$ or $\left.s_{2}=1\right\}$. At the remaining boundary $\Gamma_{D}$, i.e. the left and right side of the domain, we introduce randomized boundary conditions of the form

$$
\begin{array}{ll}
u_{D}(s)=a_{0} \cdot s_{2}+a_{1}\left(1-s_{2}\right) & s \in\left\{s \mid s_{1}=0\right\} \\
u_{D}(s)=a_{2} \cdot s_{2}+a_{3}\left(1-s_{2}\right) & s \in\left\{s \mid s_{1}=1\right\}
\end{array}
$$

with $a_{i} \sim \mathcal{U}[-0.5,0.5]$.

We model $\kappa(s)$ with a log-normally distributed random field i.e. $\kappa(s)=e^{\lambda(s)}$ where the underlying Gaussian field has a spatially constant mean $\mu_{\lambda}$ and a covariance $C_{\lambda}\left(s, s^{\prime}\right)$ function given by

$$
\mathcal{C}_{\lambda}\left(\boldsymbol{s}, \boldsymbol{s}^{\prime}\right)=\sigma_{\lambda}^{2} \cdot \exp \left(-\frac{1}{2} \frac{\left\|\boldsymbol{s}-\boldsymbol{s}^{\prime}\right\|_{2}^{2}}{l_{\lambda}^{2}}\right)
$$

The following values were used for the parameters: $\mu_{\lambda}=0.4, \sigma_{\lambda}=0.8$ and $l_{\lambda}=0.04$ or 0.15 (depending on the resolution of the FGM). The resulting random field $\kappa(s)$ exhibits significant variability with a coefficient of variation of 0.95 and the small correlation lengths necessitate fine discretizations resulting in a high-dimensional random input $\boldsymbol{x}$.

The numerical solution of the governing equations is obtained using a standard Finite Element (FE) schemes. For the purposes of our illustrations we consider the following two FE discretizations giving rise to the fine-grained (FGM) and coarse-grained (CGM) models in the previous discussion:

FGM This employs a fine(r) discretization using a regular grid of size $d_{f} \times d_{f}^{5}$. Our simulations

\footnotetext{
${ }^{5}$ The use of regular grids is pursued in order to enable the use of convolutional neural networks (CNNs) (LeCun et al., 1999; Krizhevsky et al., 2012) for the parameterized densities, enabling a parsimonious description of a complex hierarchy of features. We note that expressing physically meaningful spatio-(temporal) features on possibly non-regular and unstructured domains is a challenge in itself, but not the subject of this investigation. As such we have chosen to constrain ourselves to the representation of the random field on a regular grid, which enables the use of methods that have reached maturity due to their extensive use in computer vision.
} 
are based on $d_{f}=32$ (for $l_{\lambda}=0.15$ ) and $d_{f}=64$ (for $l_{\lambda}=0.04$ ) giving rise to $\operatorname{dim}(\boldsymbol{x})=$ $\operatorname{dim}(\boldsymbol{y})=1024$ and 4096 respectively. The random field $\kappa(s)$ is discretized using piecewise constant functions over each pixel and the vector $\boldsymbol{x}$ represents the value of $\kappa(s)$ at the centroid of each pixel. Hence $\operatorname{dim}(\boldsymbol{x})=d_{f}^{2}$.

In anticipation of the virtual observables that will be enforced and are discussed in more detail in section 3.3, we review here the weak form of the governing PDE which, in view of Equation (44) and the boundary conditions in Equation (46) and Equation (47) becomes:

$$
-\int_{\Omega} \nabla_{s} w \cdot J d s-\int_{\Omega} w f d s=0
$$

or upon making use of the constitutive equation (45)

$$
\int_{\Omega} \nabla_{s} w \cdot \kappa \nabla_{s} u d s-\int_{\Omega} w f d s=0 .
$$

The admissible weight functions $w \in \mathcal{W}$ belong in the set $\mathcal{W}=\left\{w(\boldsymbol{s}) \mid w(\boldsymbol{s}) \in H^{1}(\Omega), w(\boldsymbol{s})=\right.$ 0 on $\left.\Gamma_{D}\right\}$. We denote by $\boldsymbol{y}$ the discretized representation of $u(s)$ with the usual FE shape functions which, upon substitution in Equation (51), and for each $w \in \mathcal{W}$ yields a residual $r_{w}: \mathbb{R}^{d_{x}} \times \mathbb{R}^{d_{y}} \rightarrow \mathbb{R}:$

$$
r_{w}(\boldsymbol{y} ; \boldsymbol{x})=0
$$

We note that depending on the choice of the weight functions $w$ (at least) six methods (i.e. collocation, sub-domain, least-squares, (Petrov)-Galerkin, moments) arise as special cases (Finlayson, 1972).

It is also well-known that the solution to this problem, as with many problems in computational physics, can be obtained by minimizing an appropriate functional which in this case reduces to the potential energy function $\mathcal{V}$ given by:

$$
\mathcal{V}=\frac{1}{2} \int_{\Omega} \kappa\left|\nabla_{s} u\right|^{2} d s-\int_{\Omega} f u d s
$$

Upon discretization, this suggests that the solution vector $\boldsymbol{y}$ can be found by minimizing $V$ i.e.:

$$
\min _{\boldsymbol{y}} V(\boldsymbol{y} ; \boldsymbol{x})
$$

where $V$ is the discretized potential energy obtained by using the discretized versions of $\kappa$ and $u$ in $\mathcal{V}$ of Equation (53).

We note that the output vector $y$ which corresponds to the discretization of $u(s)$ is of similar dimension $d_{y}=\operatorname{dim}(\boldsymbol{y})=\left(d_{f}+1\right)^{2}$ as well ${ }^{6}$ (Figure 4$)$. We do not consider the discretization error of the FGM, as our goal in this work is to predict $\boldsymbol{y}$ (i.e. the discretized solution), and as such assume it to be of sufficient accuracy.

CGM This is based on a FE solver on a coarse(r) regular grid of size $d_{c} \times d_{c}$. Analogously to the FGM, the CGM input vector $\boldsymbol{X}$ represents the property within each of the pixels and is therefore of dimension $\operatorname{dim}(\boldsymbol{X})=d_{c}^{2}$. The FE solver yields the output vector $\boldsymbol{Y}$ (which

\footnotetext{
${ }^{6}$ excluding boundary conditions
} 
FGM

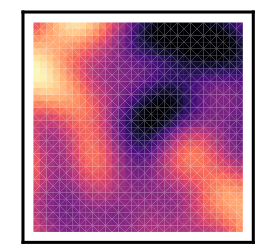

CGM $(1 \times 1)$

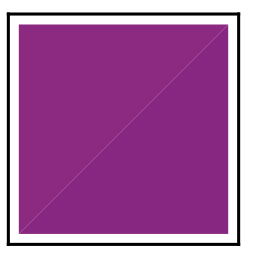

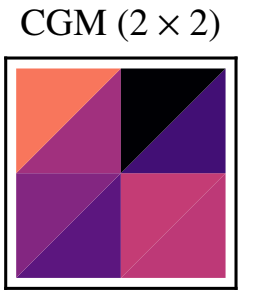
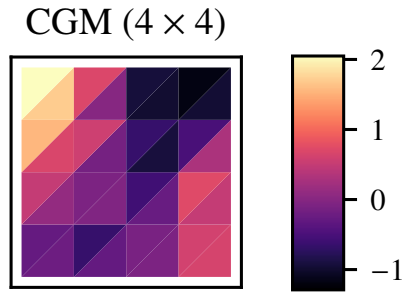

Figure 4: Comparison of a sample $\boldsymbol{x}^{(i)}$ of the discretized of the Gaussian random field $\lambda(\boldsymbol{s})$ of the FGM (left - Equation (49) with $l_{\lambda}=0.15$ ) with the (log of the posterior mean of the) corresponding $\boldsymbol{X}^{(i)}$ for three different CGM discretizations i.e. $1 \times 1,2 \times 2$ and $4 \times 4$ (The posterior means $\mathbb{E}\left[q\left(\boldsymbol{X}^{(i)}\right)\right]$ are based on $N_{l}=512$ training data). The CGMs encode effective properties $\boldsymbol{X}^{(i)}$ via the trained model density $p(\boldsymbol{X} \mid \boldsymbol{x})$. As the CGM is refined, it captures more details of the underlying FGM properties, e.g. areas in the problem domain with higher/lower conductivity $\boldsymbol{x}$ in the FGM correspond to higher/lower values of $\boldsymbol{X}$ in the CGM.

represents $u(\boldsymbol{s}))$ and is therefore of dimension $\operatorname{dim}(\boldsymbol{Y})=\left(d_{c}+1\right)^{2}$ as well ${ }^{7}$. Various values of $d_{c}$ were considered (see Figure 4) - in all cases $d_{c}<<d_{f}$ ) in order to assess the effect of the dimensionality of the CGM in the predictive estimates. We note that this particular form of the CGM was adopted for simplicity and due to the fact that boundary conditions can be readily incorporated in it rather than having to learn their effect as well (e.g. by including them in $\boldsymbol{x}, \boldsymbol{X})$. Nevertheless, any coarse-grained or reduced-order model from the vast literature on this topic can be employed instead.

\subsection{Specification of the generative model}

Given the physical problem above and the definitions of the associated input $\boldsymbol{X}, \boldsymbol{x}$ and output vectors $\boldsymbol{Y}, \boldsymbol{y}$, we provide details on the parameterization of the generative model which was generically described in section 2 . In particular, the following modeling choices were made:

(a) we employ a densely connected convolutional neural network (Huang et al., 2017) to parameterize the mean $\boldsymbol{f}\left(\boldsymbol{z} ; \boldsymbol{\theta}_{x}\right)$ as well as the input-dependent diagonal covariance matrix $\boldsymbol{S}_{\boldsymbol{x}}\left(\boldsymbol{z} ; \boldsymbol{\theta}_{x}\right)$ in Equation (17). In addition, we make use of the same architecture for the amortized encoder $q_{\Phi}(z \mid x)$ (section 2.5). More specifically, the implementation is based on a variation of the architecture proposed in (Zhu and Zabaras, 2018). The alterations refer predominantly to a reduction in the complexity and expressivity since the latent space $z$ encodes the salient features of $\boldsymbol{x}$, i.e. we only wish to retain information to the extent that it can help us in predicting effective properties by means of $p_{\boldsymbol{\theta}}(\boldsymbol{X} \mid z)$ (Equation (18)).

(b) The conditional density $\mathcal{N}\left(\boldsymbol{X} \mid \boldsymbol{g}\left(z ; \boldsymbol{\theta}_{g}\right), \boldsymbol{S}_{\boldsymbol{X}}\right)$ defined by Equation (18) relates the latent encoding $z$ to the input $\boldsymbol{X}$ of the CGM (i.e. the apparent/effective/homogenized properties). The mean vector $\boldsymbol{g}\left(z ; \boldsymbol{\theta}_{g}\right)$ depends on the latent variables $\boldsymbol{z}$ and is parameterized using a linear

\footnotetext{
${ }^{7}$ excluding boundary conditions
} 

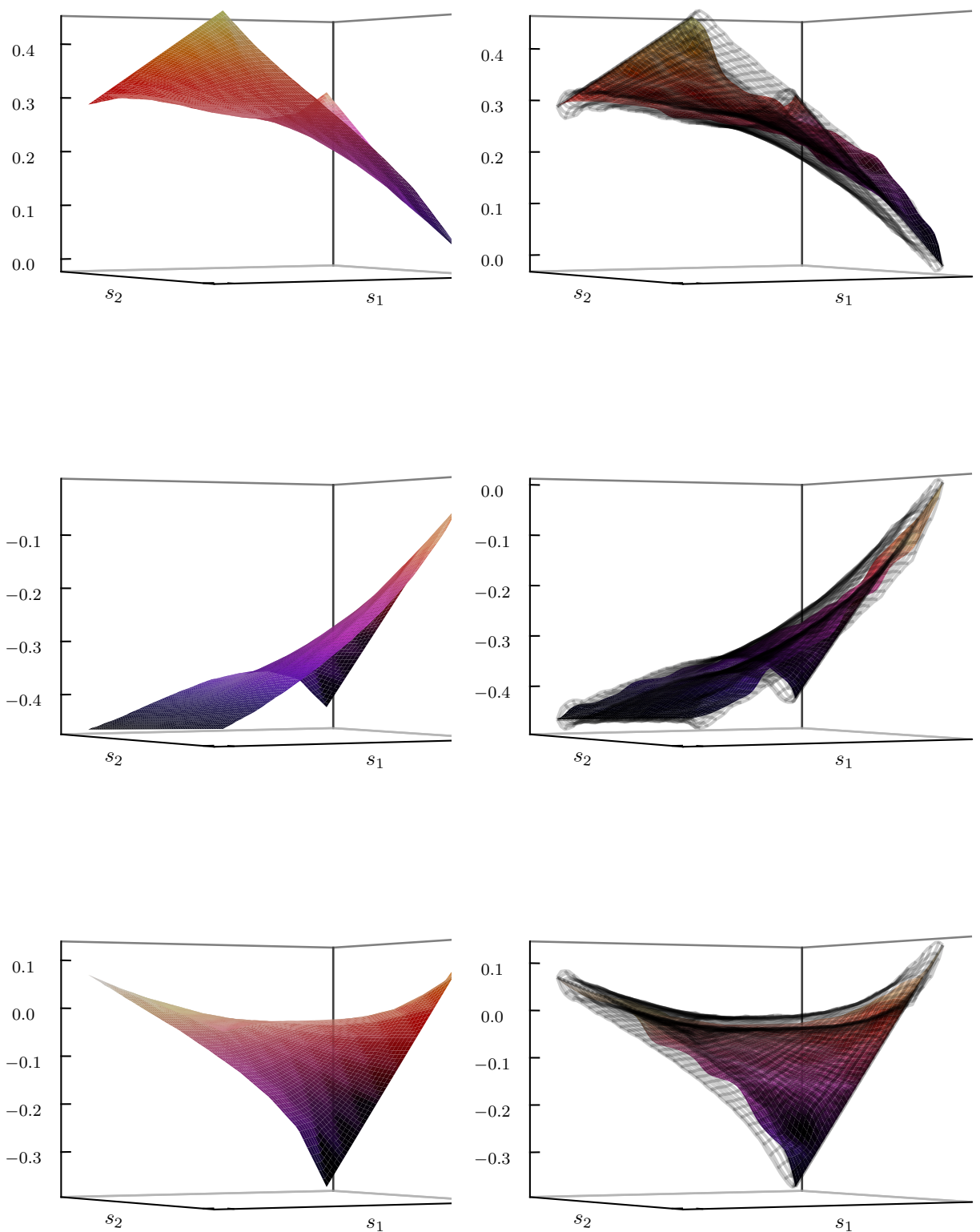

Figure 5: The left column provides examples of the mean of the posterior predictive $p(\boldsymbol{y} \mid \boldsymbol{x}, \mathcal{D})$ for various examples $x$ not seen during training. On the right we contrast this CGM based predictions with the actual solution obtained by solving the FGM (ground truth / reference), where the enveloping black mesh corresponds to the $90 \%$ confidence interval of the posterior predictive. $\left((64 \times 64) \mathrm{FGM},(8 \times 8) \mathrm{CGM}, l_{\lambda}=0.04\right)$ 
layer, i.e. $\boldsymbol{g}\left(\boldsymbol{z} ; \boldsymbol{\theta}_{g}\right)=\boldsymbol{W}_{g} \boldsymbol{z}+\boldsymbol{b}_{g}$ such that $\boldsymbol{\theta}_{g}=\left\{\boldsymbol{W}_{g}, \boldsymbol{b}_{g}\right\}$, which was found to be most robust in the low-data regime (this could be trivially expanded to a shallow feedforward neural network).

(c) For the dimension of the latent space we adopt the choice $\operatorname{dim}(z)=0.5 \cdot \operatorname{dim}(X)$. To motivate this choice, we note that the primary function of $z$ is to induce an information bottleneck which is able to retain information about effective properties $\boldsymbol{X}$. A suitable choice however will always be problem-dependent (see also section 3.7).

The general implementation of the model leverages and intertwines both Fenics (Logg et al., 2012) as well as PyTorch (Paszke et al., 2017). The CGM and its adjoint have been fully embedded within the automatic differentiation framework of PyTorch, enabling the fast and parallel solution of the CGM on the GPU (i.e. in batches).

\subsection{Virtual Observables}

Following the general discussion in section (2.2) on how domain knowledge can be introduced consistently in a probabilistic graphical model as artificial nodes (virtual observables), we discuss several types of such virtual observables $\mathcal{D}_{O}$ derived from the governing equations. We are primarily interested in those that can inexpensively augment the training data and improve the predictive ability of the trained model even though they might provide incomplete or partial pieces of information at each input query point $\boldsymbol{x}^{\left(i_{O}\right)}$ about the underlying governing equations. This property (partial information) will be reflected in the fact that most constraints we consider only carry information about a small subset of dimensions in the $\boldsymbol{y}$-space. We note that when the virtual observables $\boldsymbol{o}(\boldsymbol{y} ; \boldsymbol{x})$ are linear with respect to $\boldsymbol{y}$, then low-rank, closed-form updates for $\left\{q\left(\boldsymbol{y}^{\left(i_{O}\right)}\right)\right\}_{i_{O}=1}^{N_{O}}$ (Equation (27)) can be employed. Detailed information on these technical matters is provided in Appendix B and in the appendices referenced in the ensuing discussion.

Weighted Residuals As discussed in the previous section, the method of weighted residuals can be used to enforce the governing equations. Hence we propose using Equation (51) as constraints that are probabilistically incorporated in the proposed model as discussed in section 2.2. We note that the use of weighted residuals of PDEs has also been advocated in deterministic machine-learning loss functions (Khodayi-Mehr and Zavlanos, 2019). We consider two categories of residuals $r_{w}(\boldsymbol{y} ; \boldsymbol{x})$ based on two different types of weight functions $w$. The latter can be thought of as the lens through which the governing equations are viewed.

The first type, which we call Coarse-Grained Residuals, employs weight functions $w$ that correspond to the coarser discretization of the CGM. Due to the lower resolution of the corresponding mesh, they can be thought as enforcing the governing equations in a spatially-averaged sense. In particular and if we denote by $\boldsymbol{\Psi}(\boldsymbol{s})=\left\{\Psi_{m}(\boldsymbol{s})\right\}_{m_{1}=1}^{M_{1}}$ the vector containing the shape-function of the CGM, we consider $M_{1}$ weight functions $\left\{w_{m_{1}}\right\}_{m_{1}=1}^{M_{1}}$ of the form ${ }^{8}$ :

$$
w_{m_{1}}(s)=\Psi_{m_{1}}(s), \quad m_{1}=1, \ldots, M_{1}
$$

The second type of residuals considered and which we call Randomized Residuals are based on using $M_{2}$ radial basis-type functions as weight functions $w$ i.e.

\footnotetext{
${ }^{8}$ We always ensure these are admissible.
} 

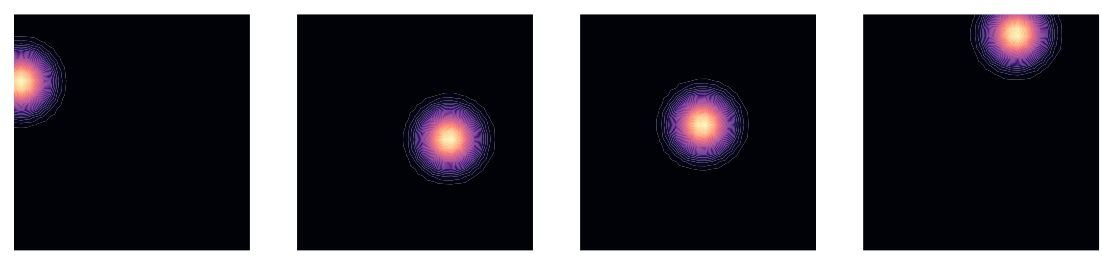

Figure 6: Illustration of 4 randomly sampled radial basis-type weight functions (Eq. (56)) corresponding to the Randomized Residuals. Instead of randomly sampling collocation point at which the PDE is enforced, we randomly sample Galerkin weight functions that enforce governing equations in a spatially-averaged sense.

$$
w_{m_{2}}(\boldsymbol{s})=\exp \left(-\frac{\left\|\boldsymbol{s}-\boldsymbol{s}_{0, m_{2}}\right\|^{2}}{\ell_{m_{2}}^{2}}\right), \quad m_{2}=1, \ldots, M_{2}
$$

The scale parameters $\left\{\ell_{m_{2}}\right\}_{m_{2}=1}^{M_{2}}$ were set equal to 0.1 in subsequent investigations, and the centers $\left\{\boldsymbol{s}_{0, m_{2}}\right\}_{m_{2}=1}^{M_{2}}$ are sampled uniformly over the problem domain i.e. [0,1] $]^{2}$.

In contrast to the first type of residuals, these are capable of providing more localized information and over subdomains the size of which is determined by the scale parameters $\ell_{m_{2}}$ which can be adjusted accordingly. In the extreme where $\ell_{m_{2}} \rightarrow 0$, the weight function $w_{m_{2}}$ becomes a Dirac- $\delta$ function and the corresponding constraint, a collocation-type one. The constraints associated with weighted residuals are enforced with infinite precision i.e. $\sigma_{c}=0$ in Equation (8).

Conservation (Flux) Constraint The second category of constraints that we employ can also be cast as a special case of weighted residuals, but operating instead directly on the conservation law (Equation (44)) i.e. on the flux variable $\boldsymbol{J}$ as in Equation (50). In particular, we make use of indicator functions of subdomains $\Omega_{m_{3}} \subseteq \Omega$ as weight functions $w_{m_{3}}$, i.e.:

$$
w_{m_{3}}(s)=1_{\Omega_{m_{3}}}(s), \quad m_{3}=1, \ldots, M_{3} .
$$

We note that in this case, Equation (50) reduces to:

$$
\int_{\partial \Omega_{m_{3}}} \boldsymbol{J} d \Gamma-\int_{\Omega_{m_{3}}} f d \boldsymbol{s}=0
$$

where the first integration is over the boundary of $\Omega_{m_{3}}$. The subdomains $\Omega_{m_{3}}$ are selected to coincide with the finite elements of the CGM (Figure 4). The flux $\boldsymbol{J}$ is computed using the constitutive law in Equation (45) from the discretized solution vector $\boldsymbol{y}$. Even though the spatial resolution of the weight functions is analogous to the ones in the Coarse-Grained Residuals above, the information the residuals of Equation (58) provide is of a different physical nature. Since not even the FGM satisfies such flux constraint perfectly, we learn the precision $\sigma_{c}^{-2}$ (Equation (8)) with which these constraints are enforced by introducing a prior that promotes larger values (Appendix A). This is analogous to the well-known 
Automatic Relevance Determination (ARD, (Bishop, 2006)) on the associated constraints.

Energy The final constraint that we make use of pertains to the type presented in Equation (10) (section 2.2) where the actual potential energy (Equation (54)) is employed. In contrast to the other constraints discussed, this provides complete information at each input query point, i.e. by minimizing $V$ which implies fully enforcing the corresponding virtual observable, one can perfectly determine the solution vector $\boldsymbol{y}$. This precludes low-rank updates and makes the incorporation of this constraint more expensive. We provide details on how $\left\{q\left(\boldsymbol{y}^{\left(i_{O}\right)}\right)\right\}_{i_{O}=1}^{N_{O}}$ is updated using stochastic second-order optimization in Appendix D.

\subsection{Predictive performance and the effect of $N_{l}$}

In the simplest scenario, the model is given access solely to a set of labeled data $\mathcal{D}_{l}=\left\{\boldsymbol{x}^{\left(i_{l}\right)}, \boldsymbol{y}^{\left(i_{l}\right)}\right\}_{i_{l}=1}^{N_{l}}$ (i.e. $N_{u}=N_{O}=0$ ). In the following we demonstrate as a baseline that the model generalizes well in the Small labeled Data regime, owing to the use of the information-bottleneck variables $z$ as well as the CGM.

As observed in Figure 7, the model achieves very high scores with only $N_{l}=128$ labeled data in terms of the $R^{2}$ (the largest possible value of $R^{2}$ is 1 ) and $N_{l}=64$ in terms of the $L S$ score. We observe that further increase of $N_{l}$ results in minimal if not negligible improvement i.e. the model has saturated. While alterations in the neural networks involved can be expected to change the particular values, we note that the saturation effect is a consequence of the limited capacity of the CGM which lies at the center of the model proposed. That is, for a given a CGM with the optimal values for its parameters, we can only predict the FGM output $y$ up to a certain level of detail. Hence even if infinite (labeled) data were available, the predictive scores of the model would not improve further and the remaining pieces would be enveloped by the predictive uncertainty (see Figure 5). On the other hand, if the CGM was removed and was substituted by a more expressive (and with more parameters) black-box model (e.g. another neural net), its predictive performance would not be as high with so few labeled data but would continue to increase (as much as its capacity would allow) with increasing $N_{l}$. This saturation effect arising from the CGM has also been observed in the discriminative model proposed in (Grigo and Koutsourelakis, 2019b) where procedures for the adaptive refinement of the CGM were proposed. These were driven by the ELBO $\mathcal{F}$, which provides a natural score function for each model, but were not pursued in this work. 


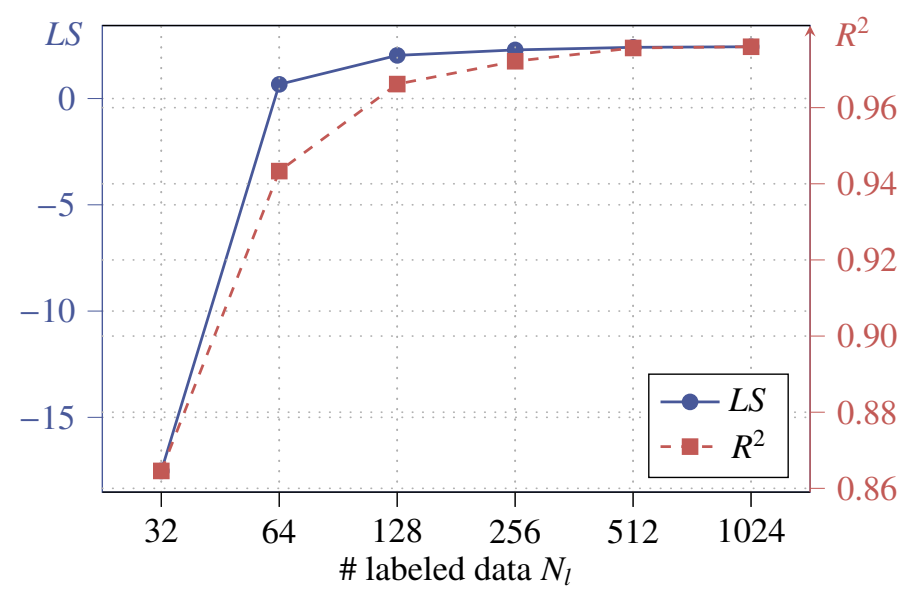

Figure 7: Predictive performance in terms of the $R^{2}$ and $L S$ metrics as a function of the number of labeled data points $N_{l}\left(N_{u}=N_{O}=0\right)$. Results have been averaged by repeatedly training the model on resampled data.

\subsection{Effect of the amount and type of virtual observables}

In the following, we demonstrate the benefits of the inclusion of virtual observables to the predictive performance of the proposed model. In order to quantify this benefit, we consider the posterior predictive density $p\left(\boldsymbol{y} \mid \boldsymbol{x}, \mathcal{D}_{l}, \mathcal{D}_{O}\right)$ (section 2.6) as a function of labeled data $\mathcal{D}_{l}$ as well as of the virtual observables $\left.\mathcal{D}_{O}=\left\{\boldsymbol{x}^{(i)}, \hat{\boldsymbol{o}}^{(i)}\right)\right\}_{i=1}^{N_{O}}$. We omit in these experiments, unlabeled data $\mathcal{D}_{u}$ (i.e. $N_{u}=0$ ), the effect of which will be examined in section 3.6. In particular, we examine the improvement in the predictive performance, i.e. in the metrics $R^{2}$ and $L S$ (section 2.6.1), of the three baseline models (for $N_{O}=0$ ) corresponding to the following number of labeled data i.e.:

$$
N_{l}=\{16,32,64\}
$$

when $N_{O}$ virtual observables are added, where:

$$
N_{O}=\{32,64,128,196,256\}
$$

Furthermore, we examine the effect of the different types of virtual observables by considering the following three categories:

- CGR: At each input query point $\boldsymbol{x}^{\left(i_{0}\right)}, M_{1}=25$ Coarse-Grained Residuals (Equation (55)) are observed .

- Hybrid: At each input query point $x^{(i o)}$ the CGR $\left(M_{1}=25\right)$, a set of Randomized weighted residuals $\left(M_{2}=60\right.$, Equation (56)) and the conservation of flux $\left(M_{3}=32\right.$, Equation (57)) are observed.

- Energy: At each input query point $\boldsymbol{x}^{\left(i_{o}\right)}$ the potential energy is observed.

we report on results in Figure Figure 8, where the left column depicts the evolution of the $R^{2}$ and $L S$ for different values of $N_{O}$ and for virtual observables of the CGR type. One can readily 
observe that, for all three $N_{l}$ values (i.e. number of labeled data), the introduction of the domainknowledge in the form of these residual-type constraints leads to a significant improvement of the model's predictive accuracy. Furthermore, with the virtual observables introduced, one can attain with only $N_{l}=16$, scores that in Figure 7 required $N_{l}=512$ labeled data i.e. a significant reduction in the number of times the FGM needs to be solved. As one would perhaps expect, the gains from the virtual observables are more pronounced for small numbers of labeled data i.e. when the model still struggles to generalize based on the too few labeled data points and therefore has more room to improve. Despite the fact that these virtual observations $\hat{\boldsymbol{o}} \in \mathbb{R}^{32}$ only provide partial information, the model is still able to leverage this to improve upon its predictive performance.

In the right column of Figure 8 we expand upon these results by considering different types of virtual observables and by quantifying the impact of their informational content on the model's predictive performance. We note that the energy virtual observables have the most striking benefit which was to be expected as they provide complete information on the associated FGM output. Secondly, the Hybrid-type seems to yield a higher improvement in the model's predictive score as compared to the CGM-type. Finally in Figure 9, we provide additional details by depicting the $L S$ metric as a function of both $N_{O}$ and $N_{l}$. 
(a) $N_{l}=16 \mid \mathrm{CGR}$

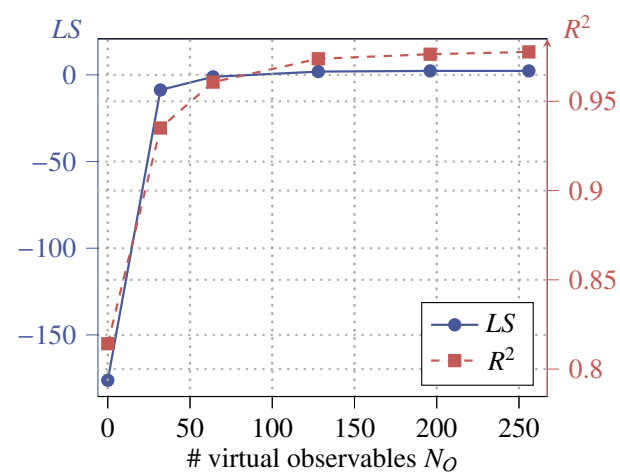

(b) $N_{l}=32 \mid \mathrm{CGR}$

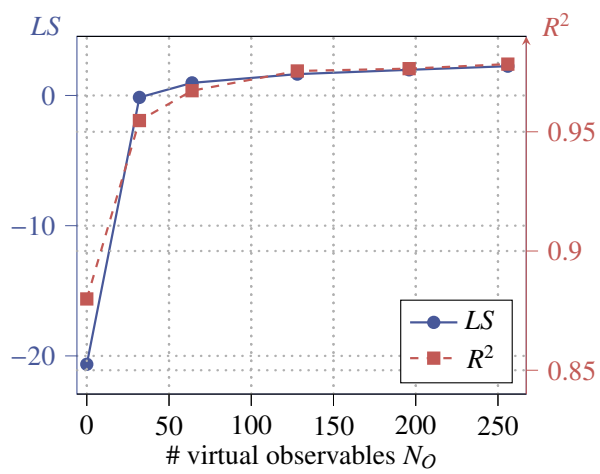

(c) $N_{l}=64 \mid \mathrm{CGR}$

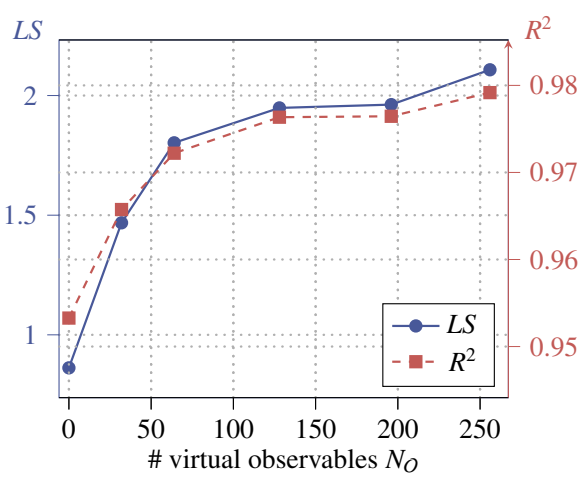

(d) $N_{l}=16 \mid$ Comparison

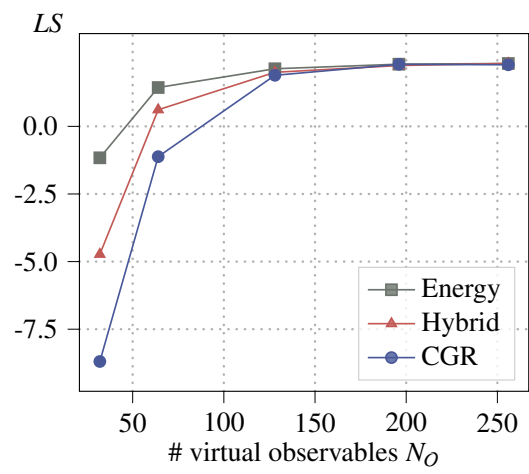

(e) $N_{l}=32 \mid$ Comparison

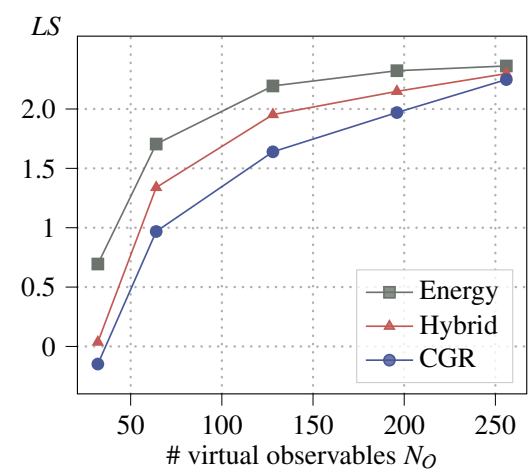

(f) $N_{l}=64 \mid$ Comparison

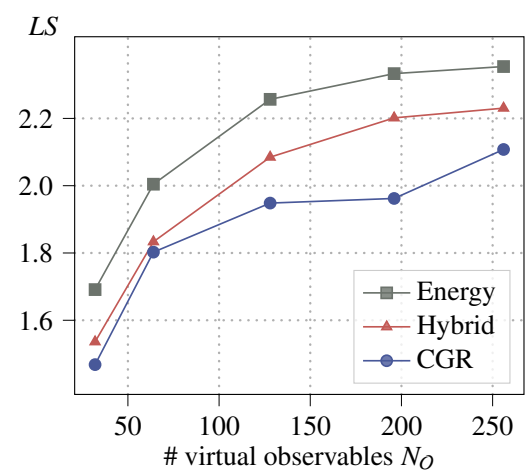

Figure 8: Left Column: Predictive performance of a model trained on $N_{l}$ labeled data, $N_{O}$ virtual observables of type CGR $\left(N_{u}=0\right)$. RIGHT CoLumn: Comparison of predictive performance in terms of the $L S$ metric with respect to 3 different types of virtual observables. The baseline performance for $N_{O}=0$ has been removed to improve clarity but the corresponding values can be found in Figure 7. Results have been averaged by repeatedly training the model on resampled data. 

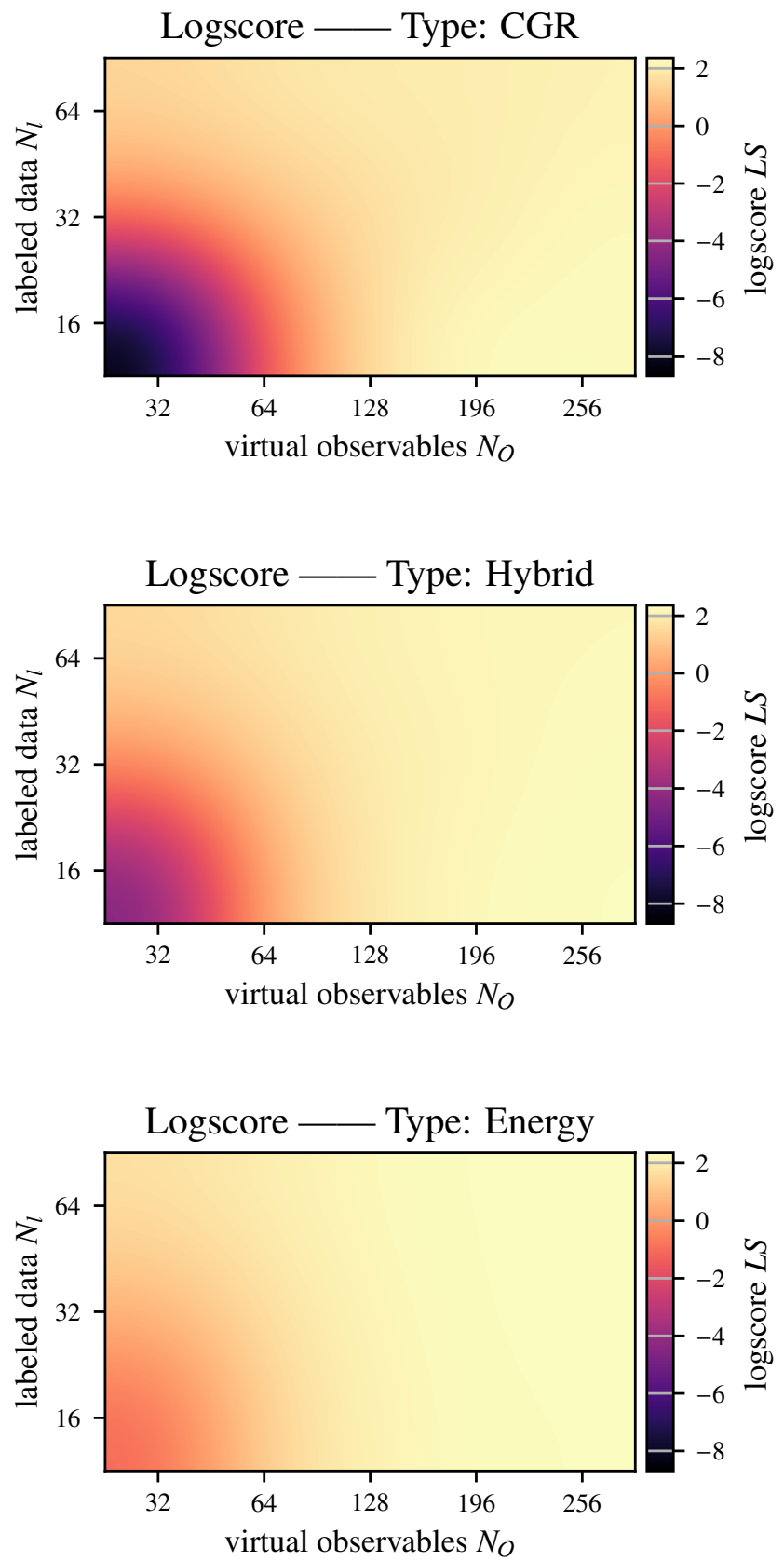

Figure 9: $L S$ Score as function of $N_{l}$ (number of labeled data) and ( $N_{O}=0$ not shown to make results more clearly visible). Results have been averaged by repeatedly training the model on resampled data. 


\subsection{Effect of unlabeled data}

In this section, we study the effect of unlabeled data $\mathcal{D}_{u}=\left\{\boldsymbol{x}^{(i)}\right\}_{i=1}^{N_{u}}$, i.e. semi-supervised learning, in the model's predictive accuracy. To this end we investigate the predictive posterior $p\left(\boldsymbol{y} \mid \boldsymbol{x}, \mathcal{D}_{u}, \mathcal{D}_{l}\right)$ as the number of unlabeled data $N_{u}$ increases. At the end of the section we consider simultaneously virtual observables $\mathcal{D}_{O}$ and assess their combined effect. mainWe reemphasize that unlabeled data are inexpensive to obtain (i.e. just inputs) and if the generative model proposed can exploit their informational context in improving its predictive ability, this would be of high utility.

In Figure 10 we present the evolution of predictive metrics $R^{2}$ and $L S$ as a function of the number of labeled data $N_{l}$ for two models. The blue line corresponds to no unlabeled data i.e. $N_{u}=0$ whereas the red line to $N_{u}=256$ such data. In both Figures the benefit of $\mathcal{D}_{u}$ can be clearly observed. The unlabeled data contribute in the identification of the lower-dimensional encoding $z$, i.e. a compressed description of the input $\boldsymbol{x}$ which in turn informs the prediction of the output $\boldsymbol{y}$ through $\boldsymbol{X}$ i.e. the CGM (Figure 2). As one can also observe, the benefit of unlabeled data decreases the higher $N_{l}$ (i.e. the number of labeled data) is. This is not unexpected as the room for improvement is smaller for higher $N_{l}$.
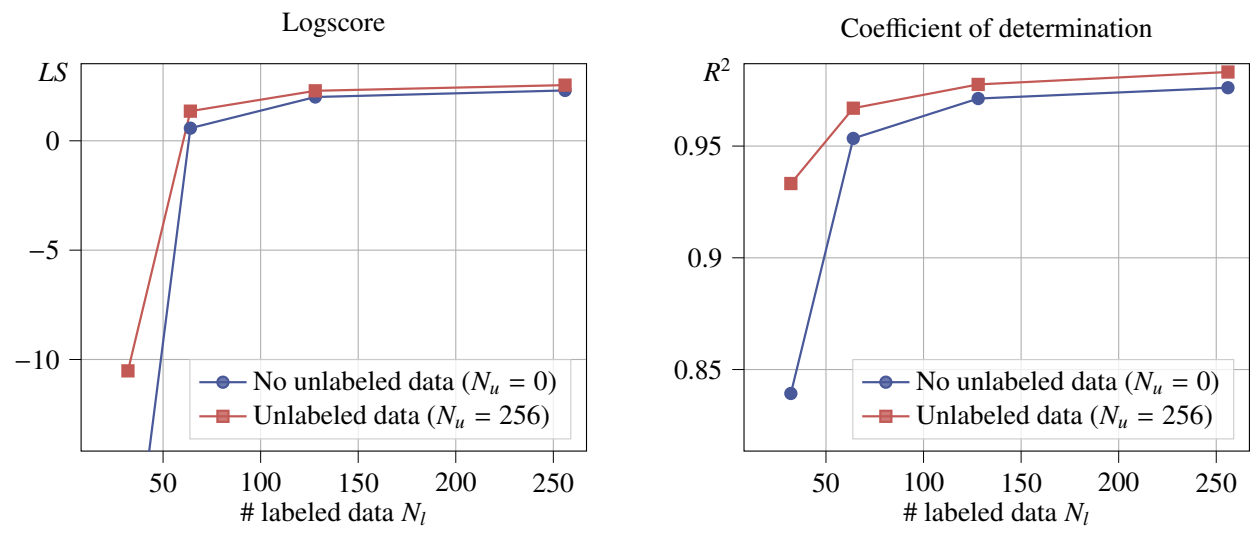

Figure 10: A model trained on a certain number of labeled data $N_{l}$ is compared to a model which in addition had access to $N_{u}=256$ unlabeled data points. The latter achieved consistently better performance. The weight afforded to unlabeled and unlabeled data in the objective (i.e. ELBO) has been normalized to be equal. Results have been averaged by repeatedly training the model on resampled data.

Figure 11 conveys similar information by varying the number of unlabeled data points while $N_{l}$ if fixed (either to $N_{l}=32$ or $N_{l}=64$ ). The improvement in the predictive performance due to addition of unlabeled data points can be clearly observed. We further note that this improvement is always less than what one would attain with additional labeled data or with virtual observables (Figure 9).

\subsection{Effect of the lower-dimensional encoding and the CGM}

In the following we provide a brief exposition of the effect of the dimension of the latent encoding $\boldsymbol{z}$ and the state variables $\boldsymbol{X}$ (and $\boldsymbol{Y}$ ) on the predictive accuracy. In Figure 12 we alter 

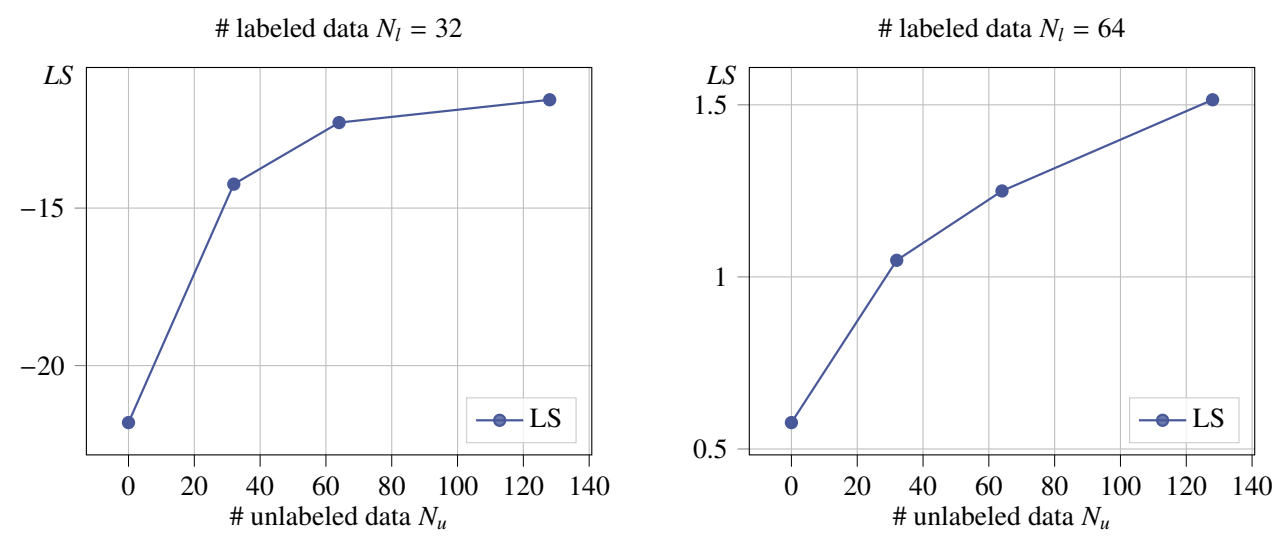

Figure 11: The predictive performance of the generative model as a function of the number of unlabeled data $N_{u}$ for $N_{l}=\{32,64\}$. Results have been averaged by repeatedly training the model on resampled data.

the dimension of the $\operatorname{dim}(z)$ and clearly observe the existence of the information bottleneck; i.e. there exists threshold for $\operatorname{dim}(z)$ up to which an improvement of the generative model is observed (for a given number of labeled data $N_{l}=256$ and $N_{u}=256$ ). After this threshold the predictive capability of the model deteriorates, since the ability to retain more information in the latent encodings is now superseded by the inability of the model to generalize well in the low-dataregime about the mappings linking the latent space to effective properties $\boldsymbol{X}$ and random field discretizations $\boldsymbol{x}$.

For the resolution of the CGM (or correspondingly the dimension of $\boldsymbol{X}$ ) one would assume to see an improvement in performance, as long as the dimension of the latent space as well as the number of datapoints afford the ability to exploit the increasing expressibility of the CGM. Here for a $(32 \times 32) \mathrm{FGM}, \operatorname{dim}(z)=32, N_{l}=512, N_{u}=512$ we illustrate in Figure 12a the improvement of the predictive performance as the discretization of the CGM is increased from a $(1 \times 1)$ to a $(4 \times 4)$. Supplementing these results we showcase in Figure 4 how effective properties of a CGM change as the dimension of $\boldsymbol{X}$ increases.

\subsection{Effect of different BCs}

In the following we evaluate the predictive performance of the model in an extrapolative setting, i.e. when the model is asked to provide predictions for boundary conditions not observed during training. To this end we consider the set of boundary conditions listed in Table 1a, where the coefficients $a_{i}$ refer to the definition of a parametric Dirichlet B.C. as given in Equation (48) (for any $a_{i}$ we specify either a fixed value, or a distribution of it to be randomly sampled from).

In Table $1 \mathrm{~b}$ we report the $L S$ score obtained on a validation dataset $\left(N_{v}=256\right)$ In all cases the model was trained on $N_{l}=512$ labeled and $N_{u}=2048$ unlabeled data (with $N_{O}=0$ ) using an amortized encoder. The diagonal terms correspond to predictive scores on the same BCs as the ones used for training (interpolative), whereas the off-diagonal ones to scores obtained on different BCs than the ones used for training (extrapolative). The results indicate that the predictive performance does not significantly depend upon the type of boundary condition the model has been trained on, i.e. the predictive performance in Table $1 \mathrm{~b}$ only varies marginally 


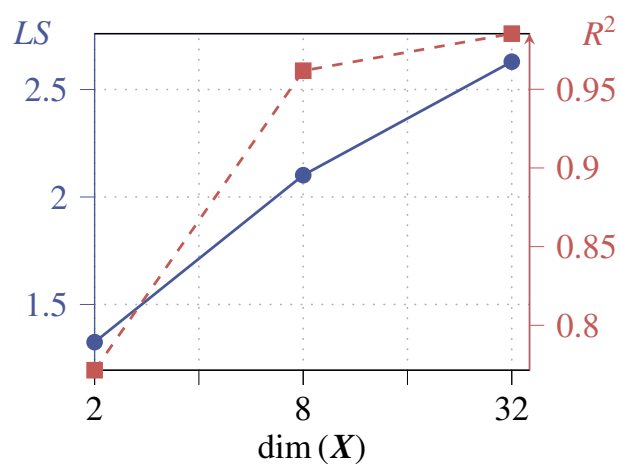

(a) Predictive performance as a function of $\operatorname{dim}(\boldsymbol{X})\left(N_{l}=512, N_{u}=512, N_{O}=0, Q=32\right)$. Results have been averaged by repeatedly training the model on resampled data.

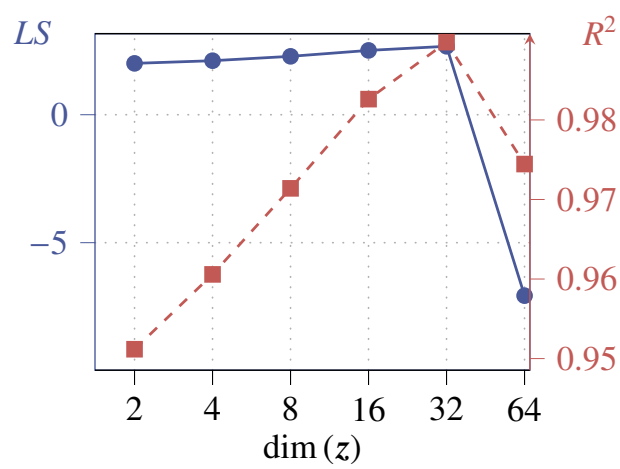

(b) Predictive performance as a function of the dimension of the latent space dimension $Q=$ $\operatorname{dim}(z)$; bottleneck occurs after $\operatorname{dim}(z)=32$ $\left(\mathrm{CGM}=(4 \times 4), N_{l}=256, N_{u}=256\right)$. Results have been averaged by repeatedly training the model on resampled data.

Figure 12: Effect of the dimension of the latent encoding $\boldsymbol{z}$ and $\boldsymbol{X}$ on the predictive performance

Boundary Conditions

\begin{tabular}{rrrrr}
\hline & $\mathrm{A}$ & $\mathrm{B}$ & $\mathrm{C}$ & $\mathrm{D}$ \\
\hline \hline$a_{0}$ & 0 & 1 & $\mathcal{U}(-0.5,0.5)$ & 0 \\
$a_{1}$ & 0 & 1 & 0 & $\operatorname{Beta}(2,5)$ \\
$a_{2}$ & 1 & 0 & 0 & $-\operatorname{Beta}(2,5)$ \\
$a_{3}$ & 1 & 0 & $\mathcal{U}(-0.5,0.5)$ & 0 \\
\hline
\end{tabular}

(a)

\section{Logscore $L S$}

\begin{tabular}{r||c|c|c|c}
$\frac{\text { prediction on }}{\text { trained on }}$ & $\mathrm{A}$ & $\mathrm{B}$ & $\mathrm{C}$ & $\mathrm{D}$ \\
\hline \hline $\mathrm{A}$ & 1.30 & 1.30 & 2.61 & 2.34 \\
\hline $\mathrm{B}$ & 1.40 & 1.40 & 2.64 & 2.39 \\
\hline $\mathrm{C}$ & 1.26 & 1.24 & 2.75 & 2.30 \\
\hline $\mathrm{D}$ & 1.17 & 1.13 & 2.44 & 2.42
\end{tabular}

(b)

Table 1: (a) Different BCs considered, and (b) Predictive performance $L S$ score obtained when training a model under the BCs indicated by the row and tested on the BCs indicated by the column.

across a column (BC used for training), and the variation is mostly determined (see row-wise), on which kind of boundary conditions we wish to make predictions.

\subsection{Application: Uncertainty Propagation}

As mentioned earlier, many-query applications represent one of the main incentives for learning such probabilistic surrogates. We consider here the case of uncertainty propagation where the goal is to compute statistics of Quantities of Interest (QoIs) associated with the output $\boldsymbol{y}$ when the input $\boldsymbol{x}$ is random with a density, say $p(\boldsymbol{x})$. In the sequel, we compare the reference solution for the density of such a scalar QoI $v(\boldsymbol{y})$ obtained by direct Monte Carlo (i.e. by generating $N_{M C}=8192$ ) samples of $\boldsymbol{x}$ and solving $N_{M C}$ times the FGM) with the marginal distribution $\tilde{p}(v \mid \mathcal{D})$ over the QoI obtained from the posterior predictive as follows: 


$$
\tilde{p}(v \mid \mathcal{D})=\iint \delta(v-v(\boldsymbol{y})) p(\boldsymbol{y} \mid \boldsymbol{x}, \mathcal{D}) p(\boldsymbol{x}) \mathrm{d} \boldsymbol{x} \mathrm{d} \boldsymbol{y}
$$

We chose as $v(y)$ the value of the solution of the PDE at the middle of our computational domain, i.e. at $s=(0.5,0.5)$. The generative model was trained with $N_{u}=8192, N_{l}=32$ and $N_{O}=256$ and the results obtained are illustrated in Figure 13. The approximation $\tilde{p}(v \mid \mathcal{D})$ obtained from the probabilistic surrogate matches closely with the Monte Carlo reference. If we had adopted a fully Bayesian approach, i.e. if $p(\boldsymbol{\theta} \mid \mathcal{D})$ was captured beyond a point estimate, additional uncertainty bounds on the probability density function $\tilde{p}(v \mid \mathcal{D})$ could be derived (Schöberl et al., 2019). Note that the approximate marginal distribution $\tilde{p}(v \mid \mathcal{D})$ has been obtained by leveraging the amortized encoder $p_{\Phi}(z \mid x)$, such that each prediction merely requires to pass $\boldsymbol{x}$ through a neural network and to solve the CGM.

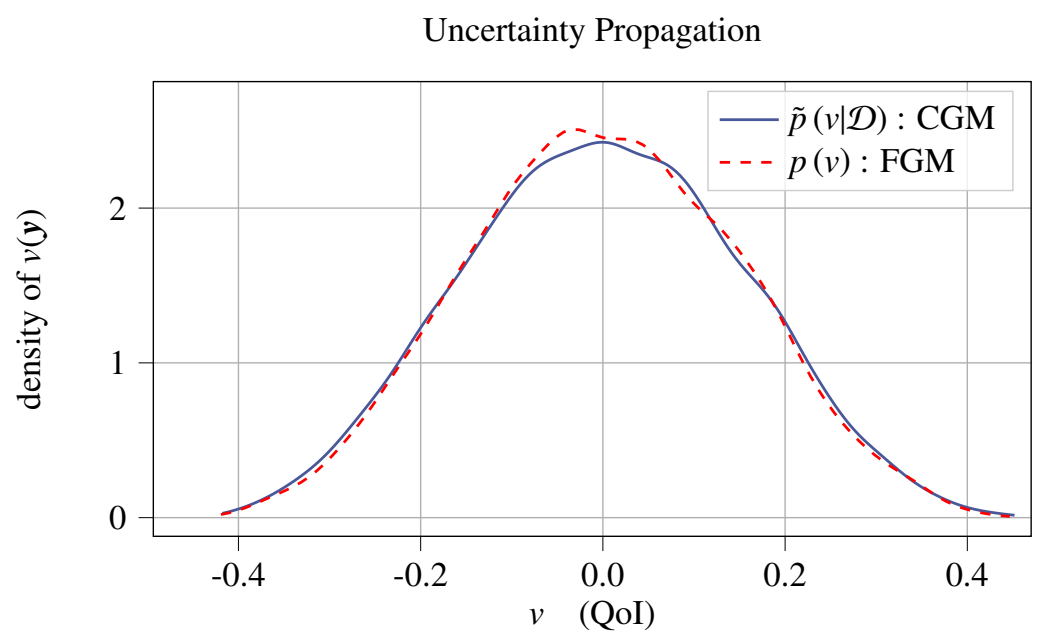

Figure 13: The predictive posterior density $p(v \mid \mathcal{D})$ over the QoI $v(\boldsymbol{y})$ as compared with the Monte Carlo reference $p(v)$ obtained with $N_{M C}=8192$ FGM solves. The model has been trained using $N_{l}=32$ (compare this with $N_{M C}$ ),$N_{u}=8192$ and $N_{O}=256$ Hybrid virtual observables (see section 3.5) and an amortized encoder was used for predictions.

\section{Conclusions}

We have proposed a generative probabilistic model for constructing surrogates for PDEs characterized by high-dimensional parametric inputs $\boldsymbol{x}$ and high-dimensional outputs $\boldsymbol{y}$. Its most important and novel characteristics are:

- it learns the joint density $p(\boldsymbol{x}, \boldsymbol{y})$ in contrast to the conditional $p(\boldsymbol{y} \mid \boldsymbol{x})$ that most discriminative models in the literature target. As a result, it can make use of unlabelled data (i.e. only inputs $\boldsymbol{x}$ ) in a semi-supervised fashion. 
- it employs a supervised dimensionality reduction scheme that identifies a set of lowerdimensional features $\boldsymbol{z}$ that are most predictive of the output $\boldsymbol{y}$. The training of this component is integrated in the overall model and is performed simultaneously with the rest.

- it employs a coarse-grained model at its core which serves as an information bottleneck between the high-dimensional inputs $\boldsymbol{x}$ and outputs $\boldsymbol{y}$. We have demonstrated how such models can be flexibly constructed by coarsening the FGM and have shown that this can lead to superior predictive performance in the Small labeled Data regime as well as under extrapolative conditions (i.e. boundary conditions not used during training).

- it makes use of domain knowledge in the form of constraints/equalities or functionals that govern the original physical problem. These are incorporated in the likelihood in a fully Bayesian fashion as virtual observables and can lead to significant performance gains while reducing further the need for expensive, labeled data.

- it yields a predictive posterior density that can be used not only for point estimates, but for quantifying the predictive uncertainty as well. The latter is most often neglected in similar efforts but it is an unavoidable consequence of any coarse-graining or dimensionalityreduction or reduced-order-modeling scheme that is trained on finite amounts of data.

The proposed modeling framework provides a fertile ground for several extensions. Apart from the obvious refinement, both in terms of breadth and depth, of the neural nets employed these improvements would involve:

- the automatic discovery of the dimension of the latent variables $z$ as well as of the CGM. In the latter case, this could involve the dimension of the state variables $\boldsymbol{X}, \boldsymbol{Y}$ as well as the model-form itself i.e. the relation between $\boldsymbol{X}$ and $\boldsymbol{Y}$. As previously mentioned, the ELBO $\mathcal{F}$ could serve as the driver for such investigations since it quantifies the plausibility of the data under a given model by balancing the quality of the fit with the model's complexity (Rasmussen and Ghahramani, 2001; Grigo and Koutsourelakis, 2019b).

- active learning in terms of unlabeled data and virtual observables. As it has been demonstrated, such data provide valuable information in improving the model. It is not necessary though that all inputs $\boldsymbol{x}$ or pairs of inputs and virtual observables $(\boldsymbol{x}, \hat{\boldsymbol{o}})$ provide the same information. A critical component in improving the overall training efficiency would be to employ active learning schemes (Kandasamy et al., 2017) in order to adaptively select the inputs and/or virtual observables (e.g. weight functions) at each step that are most informative. We note that such a scheme and in the context of a deterministic PDE-surrogate has been proposed in (Khodayi-Mehr and Zavlanos, 2019). Extensions in the probabilistic setting advocated could also make use of the ELBO in selecting from a vocabulary of options, the one that would lead to the largest increase in $\mathcal{F}$. 


\section{Appendix A. Encoding Conservation laws as equality constraints}

A wide range of PDEs imply physical conservation laws, i.e. the governing equation state that some quantity $\Psi$ is conserved and unchanging. Since this holds for any arbitrary subdomain $\Omega_{i} \subset \Omega$ and time interval we may express this in integral form (Lee and Carlberg, 2019) as

$$
\Delta \Psi_{\Omega_{i}}(t)=\frac{\mathrm{d}}{\mathrm{d} t} \int_{\Omega_{i}} \int \Psi(\boldsymbol{s}, t) \mathrm{d} \Omega_{i}+\int_{\partial \Omega_{i}} \boldsymbol{J}_{i}(\boldsymbol{s}, t) \mathrm{d}\left(\partial \Omega_{i}\right)-\int_{\Omega_{i}} f_{i}(\boldsymbol{s}, t) \mathrm{d} \Omega_{i}
$$

where $\boldsymbol{s}, \boldsymbol{J}_{i}$ and $f_{i}$ denote the spatial coordinates, (boundary) flux and source term of subdomain $\Omega_{i}$, respectively. We may introduce this physical conservation constraint into our model by introducing $o_{i}=\Delta \Psi_{\Omega_{i}}$ as a virtual observable. A virtual observable may then for instance correspond to violation of energy conservation resulting from the CGM predictions, entering into the probabilistic model by virtue of a zero-mean virtual Gaussian likelihood (e.g. $o_{i}:=\Delta \Psi_{\Omega_{i}} \sim \mathcal{N}\left(0, \tau_{i}^{-1}\right)$. For our steady-state elliptic problem with no time-dependence Equation (A.1) simplifies

$$
\Delta \Psi_{\Omega_{i}}=\int_{\partial \Omega_{i}} \boldsymbol{J}_{i}(\boldsymbol{s}) \mathrm{d} \Gamma-\int_{\Omega_{i}} f_{i}(\boldsymbol{s}) \cdot \mathrm{d} \Omega_{i}
$$

which states that the net-flow across the boundary $\partial \Omega_{i}$ must be equal to production specified by the source term (see also Equation (44)). With $u=\sum_{j=1}^{d_{y}} \varphi_{j}^{u}(s) y_{j}$ given by a Finite Element discretization of local (linear) shape functions defined on some triangulation $\mathcal{T}$ of the computational domain, Equation (A.2) results in a linear constraint, since the flux is element-wise constant (see Figure A.14), enabling us to compute

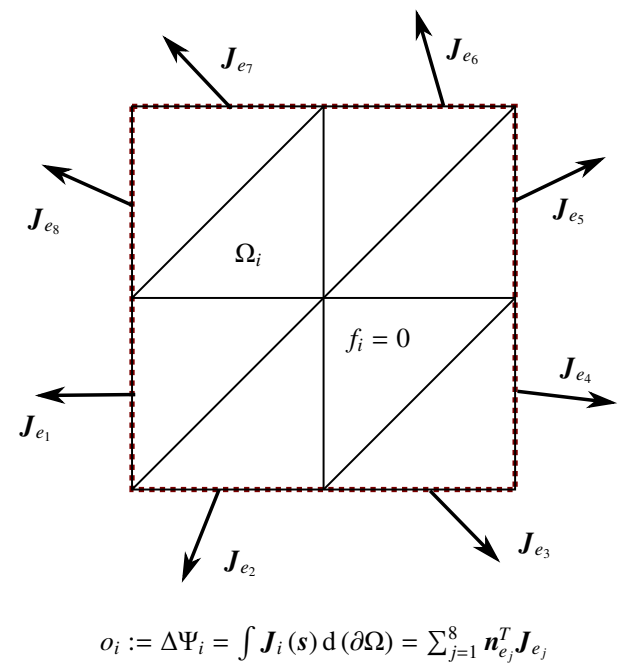

Figure A.14: If the source term $f_{i}$ associated with subdomain $\Omega_{i}$ is zero, then the integrated flux across the boundary should net to zero. The discrepancy of this flux $o_{i}:=\Delta \Psi_{\Omega_{i}}$ corresponds to a virtual observable (equality constraint) introduced as artificial node in our probabilistic graphical model. 


$$
\int_{\partial \Omega_{i}} \boldsymbol{J}(\boldsymbol{s}) \mathrm{d} \Gamma=\sum_{j=1}^{N_{e}} \boldsymbol{n}_{e_{j}}^{T} \boldsymbol{J}_{e_{j}}
$$

where the element-wise constant flux $\boldsymbol{J}_{e_{i}}=\boldsymbol{B}^{(i)} \boldsymbol{y}$ is linear in $\boldsymbol{y}$ with $\boldsymbol{B}^{(i)} \in \mathbb{R}^{2 \times d_{y}}$, and we sum over all finite elements comprising the subdomain $\Omega_{i}$ (assuming a compliant mesh). As such for the choice of $M$ subdomains $\Omega_{i}, i=1, \ldots, M$ we may define as virtual observable a vector $\boldsymbol{o}(\boldsymbol{y} ; \boldsymbol{x})$ (where the $i$-th entry corresponds to $\Delta \Psi_{\Omega_{i}}$ ) which can be expressed as

$$
o(y ; x)=\Gamma(x) y-\alpha(x)
$$

with the entries of $\boldsymbol{\Gamma}(\boldsymbol{x})$ deriving from (A.3) and $\boldsymbol{q}_{e_{i}}=\boldsymbol{B}^{(i)} \boldsymbol{y}$, while $\alpha_{i}=\int_{\Omega_{i}} f_{i}(\boldsymbol{s}) \cdot \mathrm{d} \Omega_{i}$.

\section{Appendix B. Low-Rank Mean-Field updates for virtual observables}

While in principle the entire model can be trained using stochastic variational inference ${ }^{9}$ as outlined in Algorithm 1, for linear equality constraints we are able to perform closed-form meanfield updates for $q\left(\mathcal{Y}_{O}\right)$, providing both additional insight as well as computationally efficient updates. For any ensemble of linear physical constraints enforced with a certain precision $\boldsymbol{\Lambda}$ we may write

$$
\boldsymbol{o}(\boldsymbol{y}, \boldsymbol{x}):=\boldsymbol{\Gamma}(\boldsymbol{x}) \boldsymbol{y}-\boldsymbol{\alpha}(\boldsymbol{x}) \sim \mathcal{N}\left(\mathbf{0}, \boldsymbol{\Lambda}^{-1}\right) \quad \boldsymbol{\Gamma}(\boldsymbol{x})=\left[\gamma_{1}(\boldsymbol{x})^{T}, \ldots, \gamma_{M}(\boldsymbol{x})^{T}\right] \in \mathbb{R}^{M \times d_{y}}
$$

where the entries of $\boldsymbol{\Gamma}(\boldsymbol{x})$ and $\boldsymbol{\alpha}(\boldsymbol{x})$ derive from the particular choice of constraint and the underlying physics at a query point $\boldsymbol{x}$ (see section 3.3). The precision matrix $\boldsymbol{\Lambda}=\operatorname{diag}\left(\lambda_{1}, \ldots, \lambda_{M}\right)$ is chosen diagonal, such that the set of parameters $\tau$ governing the enforcement of our constraints follows as $\tau=\left\{\lambda_{i}\right\}_{i=1}^{M}$. Given the assumed structure of the variational approximation $q_{\xi}(\boldsymbol{\theta}, \mathcal{R})$ (see Equation (27)), note that the optimal $q^{*}\left(\mathcal{Y}_{O}\right)$ follows by integrating out all other factors of $q_{\xi}$ (Bishop, 2006)

$$
\begin{aligned}
\log q^{*}\left(\boldsymbol{y}_{O}\right) & =\mathbb{E}_{\tilde{q}_{\xi}}\left[\log \left(p\left(\hat{O} \mid \boldsymbol{y}_{O}, \mathcal{X}_{O}, \boldsymbol{\Lambda}\right) p\left(\boldsymbol{y}_{O} \mid \mathfrak{X}_{O}, \boldsymbol{\theta}\right) p\left(\mathfrak{X}_{O} \mid \mathcal{Z}_{O}, \boldsymbol{\theta}\right) p\left(\mathcal{X}_{O} \mid \mathcal{Z}_{O}, \boldsymbol{\theta}\right) p\left(\mathcal{Z}_{O}\right) p(\boldsymbol{\theta})\right)\right] \\
& =\mathbb{E}_{\tilde{q}_{\xi}}\left[-\sum_{i_{O}=1}^{N_{O}}\left[\frac{1}{2}\left(\boldsymbol{y}^{\left(i_{O}\right)}-\boldsymbol{h}\left(\boldsymbol{X}^{\left(i_{O}\right)}\right)\right)^{T} \boldsymbol{S}_{\boldsymbol{y}}^{-1}\left(\boldsymbol{y}^{\left(i_{O}\right)}-\boldsymbol{h}\left(\boldsymbol{X}^{\left(i_{O}\right)}\right)\right)\right]\right] \\
& +\mathbb{E}_{\tilde{q}_{\xi}}\left[-\sum_{i_{O}=1}^{N_{O}}\left[\frac{1}{2}\left(\boldsymbol{\Gamma}\left(\boldsymbol{x}^{\left(i_{O}\right)}\right) \boldsymbol{y}-\boldsymbol{\alpha}\left(\boldsymbol{x}^{\left(i_{O}\right)}\right)\right)^{T} \boldsymbol{\Lambda}\left(\boldsymbol{\Gamma}\left(\boldsymbol{x}^{\left(i_{O}\right)}\right) \boldsymbol{y}-\boldsymbol{\alpha}\left(\boldsymbol{x}^{\left(i_{O}\right)}\right)\right)\right]\right]+\text { const. } \quad \text { (B.2) }
\end{aligned}
$$

\footnotetext{
${ }^{9}$ The required Jacobian of the virtual observables $\boldsymbol{o}(\boldsymbol{y}, \boldsymbol{x})$ in order to propagate gradients simply reduces to the well-known Gateaux derivative, and is easily (as well as cheaply and parallelizeable) obtained in most Finite Element frameworks (see e.g. Unified Form Language (Alnæs et al., 2014))
} 
where $\hat{O}=\left\{\hat{\boldsymbol{o}}_{i_{o}=1}^{N_{O}}\right.$ comprises all virtual observations and $\tilde{q}_{\xi}$ denotes all other factors of the structured mean-field approximation aside from $q\left(\boldsymbol{y}_{O}\right)$, i.e. $q_{\xi}=q\left(\mathcal{y}_{O}\right) \tilde{q}_{\xi}$. Inspecting Equation (B.2) we find that it is linear-quadratic in $\boldsymbol{y}$, which implies a Gaussian $q\left(\boldsymbol{y}^{\left(i_{O}\right)}\right)=\mathcal{N}\left(\boldsymbol{\mu}^{\left(i_{o}\right)}, \boldsymbol{\Sigma}^{\left(i_{o}\right)}\right)$ at every query point with mean and covariance implicitly defined by (for $i_{O}=1, \ldots, N_{O}$ )

$$
\begin{aligned}
\boldsymbol{\Sigma}^{\left(i_{o}\right)-1} \boldsymbol{\mu}^{\left(i_{o}\right)} & =\boldsymbol{\Gamma}\left(\boldsymbol{x}^{\left(i_{o}\right)}\right)^{T} \boldsymbol{\Lambda}\left(\boldsymbol{x}^{\left(i_{0}\right)}\right) \alpha\left(\boldsymbol{x}^{\left(i_{0}\right)}\right)+\left\langle\boldsymbol{S}_{\boldsymbol{y}}^{-1}\right\rangle\left\langle\boldsymbol{h}\left(\boldsymbol{Y}\left(\boldsymbol{X}^{\left(i_{0}\right)}\right) ; \boldsymbol{\theta}\right)\right\rangle \\
\boldsymbol{\Sigma}^{\left(i_{o}\right)^{-1}} & =\boldsymbol{\Gamma}\left(\boldsymbol{x}^{\left(i_{o}\right)}\right)^{T} \boldsymbol{\Lambda} \boldsymbol{\Gamma}\left(\boldsymbol{x}^{\left(i_{0}\right)}\right)+\left\langle\boldsymbol{S}_{\boldsymbol{y}}^{-1}\right\rangle
\end{aligned}
$$

where $\langle\cdot\rangle$ denotes an expectation with respect to all remaining factors of the variational approximation $\tilde{q}_{\xi}$. Given our model choices (Eqs.(16) - (19)), the expectation of the precision matrix $\left\langle\boldsymbol{S}_{\boldsymbol{y}}^{-1}\right\rangle$ is constrained to be diagonal while the matrix $\boldsymbol{\Gamma}\left(\boldsymbol{x}^{(i)}\right)^{T} \boldsymbol{\Lambda} \boldsymbol{\Gamma}\left(\boldsymbol{x}^{(i)}\right)$ with $\boldsymbol{\Gamma} \in \mathbb{R}^{M \times d_{y}}$ exhibits low-rank structure. This low-rank structure reflects the fact that we only have introduced partial or incomplete information, and as such the constraints are only informative for a certain (lowdimensional) subspace. It simultaneously allows us to cheaply incorporate this physical knowledge into our model, since we may exploit the low-rank structure and use the Woodbury matrix identity to obtain mean vector and covariance matrix of the Gaussians $q\left(\boldsymbol{y}^{\left(i_{0}\right)}\right)=\mathcal{N}\left(\boldsymbol{\mu}^{(i o)}, \boldsymbol{\Sigma}^{(i o)}\right)$ at a cost $O\left(M^{3}\right)$, i.e. numerical expense of updating $q\left(y^{(i)}\right)$ depends on the number of enforced constraints rather than the dimension of $\boldsymbol{y}$. Making use of the Woodbury matrix identity one finds

$$
\boldsymbol{\Sigma}^{\left(i_{O}\right)}=\left\langle\boldsymbol{S}_{\boldsymbol{y}}\right\rangle-\left\langle\boldsymbol{S}_{\boldsymbol{y}}\right\rangle \boldsymbol{\Gamma}\left(\boldsymbol{x}^{\left(i_{o}\right)}\right)^{T} \boldsymbol{\Xi}^{\left(i_{O}\right)^{-1}} \boldsymbol{\Gamma}\left(\boldsymbol{x}^{\left(i_{o}\right)}\right)\left\langle\boldsymbol{S}_{\boldsymbol{y}}\right\rangle
$$

where we have introduced the $M \times M$ matrix $\boldsymbol{\Xi}^{(i o)}=\boldsymbol{\Gamma}\left(\boldsymbol{x}^{(i o)}\right)\left\langle\boldsymbol{S}_{\boldsymbol{y}}\right\rangle \boldsymbol{\Gamma}\left(\boldsymbol{x}^{(i o)}\right)^{T}+\boldsymbol{\Lambda}^{-1}$. In the limit case of components of the diagonal precision matrix $\Lambda$ being infinite (i.e. absolute enforcement of the constraint), the result is an am improper Gaussian with rank-deficient covariance, i.e. the epistemic uncertainty of the epistemic uncertainty of the model collapses to a subspace which is completely in compliance with the enforced constraints; the update of $q\left(\boldsymbol{y}_{O}\right)$ then becomes similar to the updates of Bayesian Conjugate Gradient (BCG) (Cockayne et al., 2018), which poses the solution of a linear equation system as a problem of probabilistic inference conditionally on the observance of a set of search directions.

\section{Appendix C. Adaptively inferring finite precisions}

For some physical constraints as e.g. the flux constraint (Appendix A) it is neither plausible to assume infinite precision, nor do we a-priori know a suitable finite precision value with which to enforce the constraint. In such cases we may chose to treat the precision parameters $\tau=$ $\left\{\lambda_{m}\right\}_{m=1}^{M}$ probabilistically as well. We propose to introduce a Gamma prior $\lambda_{i} \sim \Gamma\left(\alpha_{0}^{(i)}, \beta_{0}^{(i)}\right)$ for each of the unknown precision values $\lambda^{(i)}$, or alternatively assume identical precision for all virtual observables (or subgroups thereof). For notational simplicity we discuss the latter case where all virtual observables are governed by a singular precision parameter $\lambda$

$$
\lambda \sim \frac{\beta_{0}^{\alpha_{0}}}{\Gamma\left(\alpha_{0}\right)} \lambda^{\alpha_{0}-1} \exp \left(-\beta_{0} \lambda\right)
$$


The variational approximation is extended to include $q(\lambda)$, and identically to the closed-form updates of $q\left(\boldsymbol{y}_{O}\right)$ in Appendix B the optimal variational approximation $q^{*}(\lambda)$ is to be found a Gamma distribution $\Gamma(\alpha, \beta)$, with parameters $\alpha$ and $\beta$ given by

$$
\alpha=\left(\sum_{i_{O}=1}^{N_{O}} \frac{1}{2} M\right)+\alpha_{0} \quad \beta=\frac{1}{2} \sum_{i_{O}=1}^{N_{O}} \mathbb{E}_{q\left(\boldsymbol{y}^{\left(i_{O}\right)}\right)}\left[\left\|\boldsymbol{o}\left(\boldsymbol{y}^{\left(i_{O}\right)} ; \boldsymbol{x}^{\left(i_{O}\right)}\right)\right\|_{2}^{2}\right]+\beta_{0}
$$

where $M$ the number of constraints at each query point governed by $\lambda$. For a linear constraint (B.1) and a Gaussian $q\left(\boldsymbol{y}^{\left(i_{o}\right)}\right)=\mathcal{N}\left(\boldsymbol{\mu}^{\left(i_{O}\right)}, \boldsymbol{\Sigma}^{\left(i_{O}\right)}\right)$ as given by Equation (B.3) the expectation involved in finding $\beta$ becomes tractable; otherwise they can be cheaply estimated using Monte Carlo. For the Gamma prior we chose $\alpha_{0}=\beta_{0}=10^{-6}$.

\section{Appendix D. Stochastic Second Order Optimization for the energy-based virtual observ- ables}

The introduction of the energy as a virtual observable at $N_{O}$ query point differs from the other constraints we considered, since in contrast to $M<<d_{y}$ equality constraints it fully summarizes all the information about the governing equations. Specifically, for a Finite Element discretization of the linear elliptic PDE given by $\boldsymbol{K}(\boldsymbol{x}) \boldsymbol{y}=\boldsymbol{f}(\boldsymbol{x})$, the energy can be expressed in discretized form as

$$
V\left(\boldsymbol{y}^{\left(i_{O}\right)}, \boldsymbol{x}^{\left(i_{O}\right)}\right)=\frac{1}{2} \boldsymbol{y}^{\left(i_{O}\right)^{T}} \boldsymbol{K}\left(\boldsymbol{x}^{\left(i_{O}\right)}\right) \boldsymbol{y}^{\left(i_{O}\right)}-\boldsymbol{f}\left(\boldsymbol{x}^{\left(i_{O}\right)}\right)^{T} \boldsymbol{y}^{\left(i_{O}\right)}
$$

and we find that the minimization of the quadratic potential $V\left(\boldsymbol{y}^{\left(i_{O}\right)}, \boldsymbol{x}^{i_{O}}\right)$ is the dual problem to solving the linear equation system associated with the solution of the discretized PDE itself. The introduction of the energy similarly implies that the ELBO becomes a quadratic potential in $\boldsymbol{\mu}^{\left(i_{O}\right)}$; i.e. plausibility of the model as scored by the ELBO now depends on the energy state obtained for predictions at all $N_{O}$ query points. Following the same mean-field approach as in Appendix B, the optimal $q\left(\boldsymbol{y}^{\left(i_{O}\right)}\right)=\mathcal{N}\left(\boldsymbol{\mu}^{\left(i_{O}\right)}, \boldsymbol{\Sigma}^{\left(i_{O}\right)}\right)$ is found to be a Gaussian with mean and covariance defined by (for $i=1, \ldots, N_{O}$ )

$$
\boldsymbol{\Sigma}^{\left(i_{O}\right)^{-1}} \boldsymbol{\mu}^{\left(i_{O}\right)}=\tau \boldsymbol{f}^{\left(i_{O}\right)}+\left\langle\boldsymbol{S}_{\boldsymbol{y}}^{-1}\right\rangle\left\langle\boldsymbol{h}\left(\boldsymbol{Y}\left(\boldsymbol{X}^{\left(i_{O}\right)}\right) ; \boldsymbol{\theta}\right)\right\rangle \quad \boldsymbol{\Sigma}^{\left(i_{O}\right)^{-1}}=\left\langle\boldsymbol{S}_{\boldsymbol{y}}^{-1}\right\rangle+\tau \boldsymbol{K}\left(\boldsymbol{x}^{\left(i_{O}\right)}\right)
$$

where $\tau$ is a precision or tempering parameter which governs the weight given to the virtual observables - for the limit case of $\tau$ approaching infinity, the belief about $\boldsymbol{y}^{i_{O}}$ will entirely depend on the energy state and becomes independent of the probabilistic surrogate. In contrast to the enforcement of $M<<d_{y}$ equality constraint, the precision matrix $\boldsymbol{\Sigma}^{\left(i_{O}\right)^{-1}}$ is sparse but exhibits full-rank structure, precluding the possibility to perform low-rank updates. As such the maximization of the evidence lower bound as a quadratic potential w.r.t. $\boldsymbol{\mu}^{\left(i_{O}\right)}$ on first glance appears to be the dual problem to solving the linear PDE itself if no amortization is applied. Note however that

- the maximization of the ELBO defines a simplified transfer problem since $\operatorname{cond}\left(\tau \boldsymbol{K}\left(\boldsymbol{x}^{\left(i_{O}\right)}\right)+\right.$ $\left.\left\langle\boldsymbol{S}_{\boldsymbol{y}}^{-1}\right\rangle\right) \leq \operatorname{cond}\left(\boldsymbol{K}\left(\boldsymbol{x}^{\left(i_{0}\right)}\right)\right)$, i.e. the probabilistic surrogate implicitly acts as a preconditioner. 
When optimizing the evidence lower bound we merely use the energy to correct the predictions of the surrogate and to pull them gradually in the right direction, instead of solving the PDE from scratch. This suggests an approach where one slowly tempers $\tau$ during training

- knowledge is transferred and mediated by the probabilistic model, as opposed to solving $N_{O}$ entirely disjoint problems

- we are not intrinsically interested in $q(\boldsymbol{y})$ but only to the extend to which it is able to inform our probabilistic surrogate, (i.e. learn the parameters $\boldsymbol{\theta}$ of the generative model). As such, due to the inherent irreducible error introduced by the CGM, beyond a certain point there is no benefit in increasing $\tau$, which can be seen to correspond to the tolerance parameter of iterative solvers

Despite this it has to be noted that the incorporation of this inequality constraint is comparably much more expensive. Since we want to avoid solving the equation system implied by Equation (D.2) directly, we constrain the covariance matrix $\boldsymbol{\Sigma}^{\left(i_{o}\right)}$ of the variational approximation $q\left(\boldsymbol{y}^{\left(i_{o}\right)}\right)=\mathcal{N}\left(\boldsymbol{\mu}^{\left(i_{o}\right)}, \boldsymbol{\Sigma}^{\left(i_{o}\right)}\right)$ to be diagonal and chose to optimize $\mathcal{F}$ iteratively with respects to the parameters of $q\left(\boldsymbol{y}^{\left(i_{o}\right)}\right)$ using second order stochastic optimization. Here we use randomized Newton (Gower and Richtárik, 2015; Gower et al., 2019), which can be seen to iteratively updates parameters such that the iterates are as close as possible in the L2 norm, while simultaneously forcing the error to be zero with respect to a randomly sampled subspace (see sketching-viewpoint of (Gower and Richtárik, 2015)). 


\section{References}

1. Koutsourelakis, P.S., Zabaras, N., Girolami, M.. Special Issue: Big data and predictive computational modeling. Journal of Computational Physics 2016;321:1252-1254. URL: http://www.sciencedirect.com/science/ article/pii/S0021999116001807. doi:10.1016/j.jcp.2016.03.028.

2. Marcus, G., Davis, E.. Rebooting AI: Building Artificial Intelligence We Can Trust. Pantheon; 2019.

3. Stewart, R., Ermon, S.. Label-free supervision of neural networks with physics and domain knowledge. In: Thirty-First AAAI Conference on Artificial Intelligence. 2017:

4. Koutsourelakis, P.S.. Stochastic upscaling in solid mechanics: An excercise in machine learning. Journal of Computational Physics 2007;226(1):301-325.

5. Ghanem, R.G., Spanos, P.D.. Stochastic Finite Elements: A Spectral Approach. New York: Springer; 1991. URL: http://cds.cern.ch/record/1622736.

6. Xiu, D., Karniadakis, G.. The Wiener-Askey Polynomial Chaos for Stochastic Differential Equations. SIAM Journal on Scientific Computing 2002a;24(2):619-644. URL: https : //doi .org/10.1137/S1064827501387826. doi:10. $1137 /$ S1064827501387826.

7. Xiu, D., Hesthaven, J.. High-Order Collocation Methods for Differential Equations with Random Inputs. SIAM Journal on Scientific Computing 2005;27(3):1118-1139. URL: https://doi.org/10.1137/040615201. doi:10.1137/ 040615201.

8. Ma, X., Zabaras, N.. An adaptive hierarchical sparse grid collocation algorithm for the solution of stochastic differential equations. Journal of Computational Physics 2009;228(8):3084 - 3113. URL: http: //www . sciencedirect . com/ science/article/pii/S002199910900028X. doi:https://doi.org/10.1016/j.jcp.2009.01.006.

9. Lin, G., Tartakovsky, A.. An efficient, high-order probabilistic collocation method on sparse grids for three-dimensional flow and solute transport in randomly heterogeneous porous media. Advances in Water Resources 2009;32(5):712 722. URL: http://www.sciencedirect.com/science/article/pii/S0309170808001632. doi:https:// doi.org/10.1016/j.advwatres. 2008.09.003; dispersion in Porous Media.

10. Torquato, S., Lu, B.. Chord-length distribution function for two-phase random media. Phys Rev E 1993;47:2950-2953. URL: https://link.aps.org/doi/10.1103/PhysRevE.47.2950. doi:10.1103/PhysRevE.47.2950.

11. Hesthaven, J., Rozza, G., Stamm, B.. Certified Reduced Basis Methods for Parametrized Partial Differential Equations. Springer Briefs in Mathematics; Springer International Publishing; 2016. ISBN 978-3-319-22469-5. URL: //www . springer.com/de/book/9783319224695.

12. Quarteroni, A., Manzoni, A., Negri, F.. Reduced Basis Methods for Partial Differential Equations. An Introduction. La Matematica per il 3+2. 92; Springer International Publishing; 2016. URL: http://infoscience.epfl.ch/ record/218966. doi:10.1007/978-3-319-15431-2.

13. Rowley, C.W., Colonius, T., Murray, R.M.. Model reduction for compressible flows using POD and Galerkin projection. Physica D: Nonlinear Phenomena 2004;189(1):115 - 129. URL: http://www.sciencedirect.com/science/ article/pii/S0167278903003841. doi:https://doi.org/10.1016/j.physd.2003.03.001.

14. Guo, M., Hesthaven, J.. Reduced order modeling for nonlinear structural analysis using gaussian process regression. Computer Methods in Applied Mechanics and Engineering 2018a;341:807 - 826. URL: http://www.sciencedirect.com/science/article/pii/S0045782518303487. doi:https://doi. org/10.1016/j.cma.2018.07.017.

15. Hesthaven, J., Ubbiali, S.. Non-intrusive reduced order modeling of nonlinear problems using neural networks. Journal of Computational Physics 2018;363:55 - 78. URL: http://www.sciencedirect.com/science/article/pii/ S0021999118301190. doi:https://doi.org/10.1016/j.jcp.2018.02.037.

16. Kani, J.N., Elsheikh, A.H.. Dr-rnn: A deep residual recurrent neural network for model reduction. arXiv preprint arXiv:170900939 2017;.

17. Wang, Q., Ripamonti, N., Hesthaven, J.S.. Recurrent neural network closure of parametric pod-galerkin reduced-order models based on the mori-zwanzig formalism. Journal of Computational Physics 2020;:109402.

18. Lee, K., Carlberg, K.T.. Model reduction of dynamical systems on nonlinear manifolds using deep convolutional autoencoders. Journal of Computational Physics 2020;404:108973.

19. Rasmussen, C., Williams, C.. Gaussian Processes for Machine Learning. Adaptive Computation and Machine Learning; Cambridge, MA, USA: MIT Press; 2006.

20. Bilionis, I., Zabaras, N., Konomi, B.A., Lin, G.. Multi-output separable Gaussian process: Towards an efficient, fully Bayesian paradigm for uncertainty quantification. Journal of Computational Physics 2013a;241:212 - 239. URL: http://www.sciencedirect.com/science/article/pii/S0021999113000417. doi:https: //doi.org/10.1016/j.jcp.2013.01.011.

21. Bilionis, I., Zabaras, N.. Bayesian Uncertainty Propagation Using Gaussian Processes. Cham: Springer International Publishing. ISBN 978-3-319-12385-1; 2017:555-599. URL: https://doi.org/10.1007/ 978-3-319-12385-1_16. doi:10.1007/978-3-319-12385-1_16.

22. O'Hagan, A., Kennedy, M.. Predicting the output from a complex computer code when fast approximations are 
available. Biometrika 2000;87(1):1-13. URL: https://dx.doi.org/10.1093/biomet/87.1.1. doi:10.1093/ biomet/87.1.1.

23. Koutsourelakis, P.S.. Accurate Uncertainty Quantification Using Inaccurate Computational Models. SIAM Journal on Scientific Computing 2009;31(5):3274-3300. URL: https://doi.org/10.1137/080733565. doi:10.1137/ 080733565.

24. Raissi, M., Perdikaris, P., Karniadakis, G.E.. Inferring solutions of differential equations using noisy multi-fidelity data Journal of Computational Physics 2017a;335:736 - 746. URL: http://www.sciencedirect.com/science/ article/pii/S0021999117300761. doi:https://doi.org/10.1016/j.jcp.2017.01.060.

25. Perdikaris, P., Venturi, D., Royset, J.O., Karniadakis, G.E.. Multi-fidelity modelling via recursive co-kriging and Gaussian-Markov random fields. Proceedings of the Royal Society A: Mathematical, Physical and Engineering Sciences 2015;471(2179):20150018. URL: https://royalsocietypublishing.org/doi/abs/10.1098/rspa. 2015.0018. doi:10.1098/rspa.2015.0018.

26. Yang, X., Tartakovsky, G., Tartakovsky, A.. Physics-Informed Kriging: A Physics-Informed Gaussian Process Regression Method for Data-Model Convergence. arxiv e-print 2018;URL: https ://arxiv . org/pdf/1809.03461 .pdf.

27. Lee, S., Dietrich, F., Karniadakis, G., Kevrekidis, I.. Linking Gaussian Process regression with data-driven manifold embeddings for nonlinear data fusion. arxiv e-print 2018;URL: https : //arxiv.org/pdf/1812.06467.pdf.

28. Tipireddy, R., Tartakovsky, A.. Physics-informed Machine Learning Method for Forecasting and Uncertainty Quantification of Partially Observed and Unobserved States in Power Grids. arxiv e-print 2018;URL: https: //arxiv.org/pdf/1806.10990.pdf.

29. Guo, M., Hesthaven, J.S.. Reduced order modeling for nonlinear structural analysis using gaussian process regression. Computer methods in applied mechanics and engineering 2018b;341:807-826.

30. LeCun, Y., Bengio, Y., Hinton, G.. Deep learning. Nature 2015;521(7553):436-444. URL: http://www . nature . com/nature/journal/v521/n7553/full/nature14539.html. doi:10.1038/nature14539.

31. Goodfellow, I., Bengio, Y., Courville, A.. Deep Learning. MIT Press; 2016. http://www.deeplearningbook. org.

32. Han, J., Jentzen, A., E, W.. Solving high-dimensional partial differential equations using deep learning. Proceedings of the National Academy of Sciences 2018;115(34):8505-8510. URL: https ://www . pnas . org/content/115/34/ 8505. doi:10.1073/pnas.1718942115.

33. Zhu, Y., Zabaras, N.. Bayesian deep convolutional encoder-decoder networks for surrogate modeling and uncertainty quantification. Journal of Computational Physics 2018;366:415-447.

34. Mo, S., Zhu, Y., Zabaras, N., Shi, X., Wu, J.. Deep Convolutional Encoder-Decoder Networks for Uncertainty Quantification of Dynamic Multiphase Flow in Heterogeneous Media. Water Resources Research 2018;0(0). URL: https : //agupubs.onlinelibrary.wiley.com/doi/abs/10.1029/2018WR023528. doi:10.1029/2018WR023528.

35. Sirignano, J., Spiliopoulos, K.. DGM: A deep learning algorithm for solving partial differential equations. Journal of Computational Physics 2018;375:1339-1364. URL: http://arxiv.org/abs/1708.07469. doi:10.1016/j . jcp. 2018.08.029; arXiv: 1708.07469.

36. E, W., Yu, B.. The Deep Ritz Method: A Deep Learning-Based Numerical Algorithm for Solving Variational Problems. Communications in Mathematics and Statistics 2018;6(1):1-12. URL: https://doi.org/10.1007/ s40304-018-0127-z. doi:10.1007/s40304-018-0127-z.

37. Raissi, M., Perdikaris, P., Karniadakis, G.. Physics Informed Deep Learning (Part I): Data-driven Solutions of Nonlinear Partial Differential Equations. arxiv e-print 2017b;URL: https://arxiv.org/pdf/1711.10561.pdf.

38. Raissi, M., Karniadakis, G.E.. Hidden physics models: Machine learning of nonlinear partial differential equations. Journal of Computational Physics 2018;357:125 - 141. URL: http://www . sciencedirect . com/science/ article/pii/S0021999117309014. doi:https://doi.org/10.1016/j.jcp.2017.11.039.

39. Raissi, M., Perdikaris, P., Karniadakis, G.. Physics-informed neural networks: A deep learning framework for solving forward and inverse problems involving nonlinear partial differential equations. Journal of Computational Physics 2019;378:686 - 707. URL: http://www. sciencedirect. com/science/article/pii/S0021999118307125. doi:https://doi.org/10.1016/j.jcp.2018.10.045

40. Yang, Y., Perdikaris, P.. Conditional deep surrogate models for stochastic, high-dimensional, and multi-fidelity systems. arxiv e-print 2019;URL: https://arxiv.org/pdf/1901.04878.pdf.

41. Lagaris, I., Likas, A., Papageorgiou, D.. Neural-network methods for boundary value problems with irregular boundaries. IEEE TRANSACTIONS ON NEURAL NETWORKS 2000;11(5):1041-1049.

42. Nabian, M.A., Meidani, H.. A deep neural network surrogate for high-dimensional random partial differential equations. arXiv preprint arXiv:180602957 2018;

43. Beck, C., E, W., Jentzen, A.. Machine Learning Approximation Algorithms for High-Dimensional Fully Nonlinear Partial Differential Equations and Second-order Backward Stochastic Differential Equations. Journal of Non linear Science 2019;29(4):1563-1619. URL: https://doi.org/10.1007/s00332-018-9525-3. doi:10.1007/ s00332-018-9525-3.

44. Karumuri, S., Tripathy, R., Bilionis, I., Panchal, J.. Simulator-free solution of high-dimensional stochastic elliptic partial differential equations using deep neural networks. Journal of Computational Physics 2020;404:109120. 
45. Khodayi-Mehr, R., Zavlanos, M.M.. VarNet: Variational Neural Networks for the Solution of Partial Differential Equations. 2019. URL: https://arxiv.org/abs/1912.07443.

46. Zhu, Y., Zabaras, N., Koutsourelakis, P.S., Perdikaris, P.. Physics-constrained deep learning for high-dimensional surrogate modeling and uncertainty quantification without labeled data. Journal of Computational Physics 2019;394:5681 .

47. Frank, M., Drikakis, D., Charissis, V.. Machine-Learning Methods for Computational Science and Engineering. Computation 2020;8(1):15. URL: https://www.mdpi.com/2079-3197/8/1/15. doi:10.3390/ computation8010015.

48. Willard, J., Jia, X., Xu, S., Steinbach, M., Kumar, V.. Integrating physics-based modeling with machine learning: A survey. 2020. arXiv:2003.04919.

49. Mattheakis, M., Protopapas, P., Sondak, D., Di Giovanni, M., Kaxiras, E.. Physical Symmetries Embedded in Neural Networks. arXiv:190408991 [physics] 2020;URL: http://arxiv.org/abs/1904.08991; arXiv: 1904.08991.

50. Magiera, J., Ray, D., Hesthaven, J.S., Rohde, C.. Constraint-aware neural networks for riemann problems. Journal of Computational Physics 2020;409:109345.

51. Brunton, S., Proctor, J., Kutz, N.. Sparse identification of nonlinear dynamics (sindy). In: APS Division of Fluid Dynamics Meeting Abstracts. 2016:.

52. Long, Z., Lu, Y., Ma, X., Dong, B.. Pde-net: Learning pdes from data. arXiv preprint arXiv:171009668 2017;.

53. Felsberger, L., Koutsourelakis, P.. Physics-constrained, data-driven discovery of coarse-grained dynamics. Communications in Computational Physics 2019;25(5):1259-1301. doi:10.4208/cicp.0A-2018-0174.

54. Kaltenbach, S., Koutsourelakis, P.S.. Incorporating physical constraints in a deep probabilistic machine learning framework for coarse-graining dynamical systems. 2019. arXiv:1912.12976.

55. Bilionis, I., Zabaras, N., Konomi, B.A., Lin, G.. Multi-output separable Gaussian process: Towards an efficient, fully Bayesian paradigm for uncertainty quantification. Journal of Computational Physics 2013b;241:212 239. URL: http://www.sciencedirect.com/science/article/pii/S0021999113000417. doi:10.1016/ j.jcp. 2013.01.011.

56. Xiu, D.. Numerical methods for stochastic computations: a spectral method approach. Princeton university press; 2010.

57. Xiu, D., Karniadakis, G.E.. Modeling uncertainty in steady state diffusion problems via generalized polynomial chaos. Computer methods in applied mechanics and engineering 2002b;191(43):4927-4948.

58. Grigo, C., Koutsourelakis, P.S.. Bayesian Model and Dimension Reduction for Uncertainty Propagation: Applications in Random Media. SIAM/ASA Journal on Uncertainty Quantification 2019a;7(1):292-323. URL: https ://epubs . siam.org/doi/abs/10.1137/17M1155867. doi:10.1137/17M1155867.

59. Grigo, C., Koutsourelakis, P.S.. A physics-aware, probabilistic machine learning framework for coarse-graining high-dimensional systems in the Small Data regime. Journal of Computational Physics 2019b;397:108842. URL: http://www.sciencedirect.com/science/article/pii/S0021999119305261. doi:10.1016/j.jcp.2019.05.053.

60. Chapelle, O., Schölkopf, B., Zien, A.. Semi-supervised learning. IEEE Transactions on Neural Networks 2009;20(3). doi:10.1109/TNN.2009.2015974

61. Kingma, D.P., Mohamed, S., Rezende, D.J., Welling, M.. Semi-supervised learning with deep generative models. In: Advances in neural information processing systems. 2014:3581-3589.

62. Yu, S., Yu, K., Tresp, V., Kriegel, H.P., Wu, M.. Supervised probabilistic principal component analysis. In: Proceedings of the 12th ACM SIGKDD international conference on Knowledge discovery and data mining. ACM; 2006:464-473.

63. Raissi, M., Perdikaris, P., Karniadakis, G.E.. Machine learning of linear differential equations using gaussian processes. Journal of Computational Physics 2017c;348:683-693.

64. Levine, S.. Reinforcement learning and control as probabilistic inference: Tutorial and review. arXiv preprint arXiv:180500909 2018;.

65. Ortiz, M., Stainier, L.. The variational formulation of viscoplastic constitutive updates. Computer Methods in Applied Mechanics and Engineering 1999;171(3):419-444. URL: http://www.sciencedirect.com/science/ article/pii/S0045782598002199. doi:10.1016/S0045-7825(98)00219-9.

66. Yang, Q., Stainier, L., Ortiz, M.. A variational formulation of the coupled thermo-mechanical boundary-value problem for general dissipative solids. Journal of the Mechanics and Physics of Solids 2006;54(2):401-424. URL: http: //www.sciencedirect.com/science/article/pii/S0022509605001511. doi:10.1016/j.jmps.2005.08. 010 .

67. Khoo, Y., Lu, J., Ying, L.. Solving parametric pde problems with artificial neural networks. arXiv preprint arXiv:170703351 2017;

68. Paisley, J., Blei, D., Jordan, M.I.. Variational Bayesian inference with stochastic search. In: Langford, J., Pineau, J., eds. 29th International Conference on Machine Learning (ICML). Edinburgh, UK; 2012:

69. Hoffman, M.D., Blei, D.M., Wang, C., Paisley, J.. Stochastic Variational Inference. J Mach Learn Res 2013;14(1):1303-1347. URL: http://dl .acm.org/citation. cfm?id=2502581.2502622. 
70. Blei, D.M., Kucukelbir, A., McAuliffe, J.D.. Variational inference: A review for statisticians. Journal of the American Statistical Association 2017;112(518):859-877.

71. Bishop, C.M.. Pattern Recognition and Machine Learning. Springer; 2006

72. Kingma, D.P., Welling, M.. Auto-encoding variational bayes. arXiv preprint arXiv:13126114 2013;

73. Robbins, H., Monro, S.. A stochastic approximation method. The annals of mathematical statistics $1951 ;: 400-407$.

74. Kingma, D.P., Ba, J.. Adam: A method for stochastic optimization. arXiv preprint arXiv:14126980 2014;

75. Naumann, U.. The art of differentiating computer programs: an introduction to algorithmic differentiation; vol. 24. Siam; 2012.

76. Paszke, A., Gross, S., Chintala, S., Chanan, G., Yang, E., DeVito, Z., Lin, Z., Desmaison, A., Antiga, L., Lerer, A.. Automatic differentiation in pytorch 2017;

77. Zhang, D.. A coefficient of determination for generalized linear models. The American Statistician 2017;71(4):310-316.

78. LeCun, Y., Haffner, P., Bottou, L., Bengio, Y.. Object recognition with gradient-based learning. In: Shape, contour and grouping in computer vision. Springer; 1999:319-345.

79. Krizhevsky, A., Sutskever, I., Hinton, G.E.. Imagenet classification with deep convolutional neural networks. In: Advances in neural information processing systems. 2012:1097-1105.

80. Finlayson, B., ed. The method of weighted residuals and variational principles, with application in fluid mechanics, heat and mass transfer, Volume 87. New York: Academic Press; 1972. ISBN 978-0-12-257050-6.

81. Huang, G., Liu, Z., Van Der Maaten, L., Weinberger, K.Q.. Densely connected convolutional networks. In: Proceedings of the IEEE conference on computer vision and pattern recognition. 2017:4700-4708.

82. Logg, A., Mardal, K.A., Wells, G.. Automated solution of differential equations by the finite element method: The FEniCS book; vol. 84. Springer Science \& Business Media; 2012.

83. Schöberl, M., Zabaras, N., Koutsourelakis, P.S.. Predictive collective variable discovery with deep bayesian models. The Journal of chemical physics 2019;150(2):024109.

84. Rasmussen, C., Ghahramani, Z.. Occam's Razor. In: Neural Information Processing Systems 13. 2001:294-300.

85. Kandasamy, K., Schneider, J., Póczos, B.. Query Efficient Posterior Estimation in Scientific Experiments via Bayesian Active Learning. Artif Intell 2017;243(C):45-56. URL: https://doi .org/10.1016/j .artint.2016.11.002. doi:10.1016/j.artint. 2016.11.002.

86. Lee, K., Carlberg, K.. Deep conservation: A latent dynamics model for exact satisfaction of physical conservation laws. arXiv preprint arXiv:190909754 2019;

87. Alnæs, M.S., Logg, A., Ølgaard, K.B., Rognes, M.E., Wells, G.N.. Unified form language: A domain-specific language for weak formulations of partial differential equations. ACM Transactions on Mathematical Software (TOMS) 2014;40(2):1-37.

88. Cockayne, J., Oates, C., Ipsen, I., Girolami, M.. A bayesian conjugate gradient method. 2018. arXiv:1801.05242.

89. Gower, R.M., Richtárik, P.. Randomized iterative methods for linear systems. SIAM Journal on Matrix Analysis and Applications 2015;36(4):1660-1690.

90. Gower, R.M., Kovalev, D., Lieder, F., Richtárik, P.. Rsn: Randomized subspace newton. arXiv preprint arXiv:190510874 2019;. 